\title{
Facile Synthesis and Antimicrobial Evaluation of Some New Heterocyclic Compounds Incorporating a Biologically Active Sulfamoyl Moiety
}

\author{
Elham S. Darwish \\ Department of Chemistry, Faculty of Science, Cairo University, Giza 12613, Egypt \\ Correspondence should be addressed to Elham S. Darwish; elham_darwish@yahoo.com
}

Received 7 March 2014; Accepted 4 April 2014; Published 17 August 2014

Academic Editor: Valdir Cechinel Filho

Copyright (c) 2014 Elham S. Darwish. This is an open access article distributed under the Creative Commons Attribution License, which permits unrestricted use, distribution, and reproduction in any medium, provided the original work is properly cited.

\begin{abstract}
A facile and convenient synthesis of new heterocyclic compounds containing a sulfamoyl moiety suitable for use as antimicrobial agents was reported. The precursor 3-oxo-3-phenyl- $N$-(4-sulfamoylphenyl)propionamide was coupled smoothly with arenediazonium salt producing hydrazones which reacted with malononitrile or triethylorthoformate affording pyridazine and triazine derivatives, respectively. Also, the reactivity of the same precursor with DMF-DMA was followed by aminotriazole; aromatic aldehydes was followed by hydrazine hydrate, triethylorthoformate, or thiourea affording triazolo[1,5-a]pyrimidine, pyrazole, acrylamide, and dihydropyrimidine derivatives, respectively. On the other hand, treatment of the precursor propionamide with phenyl isothiocyanate and $\mathrm{KOH}$ in $\mathrm{DMF}$ afforded the intermediate salt which was treated with dilute $\mathrm{HCl}$ followed by 2-bromo1-phenylethanone affording carboxamide derivative. While the same intermediate salt reacted in situ with chloroacetone, ethyl 2-chloroacetate, 3-(2-bromoacetyl)-2H-chromen-2-one, methyl iodide, or 2-oxo- $\mathrm{N}$-phenylpropane hydrazonoyl chloride afforded the thiophene, ketene $N, S$-acetal, and thiadiazole derivatives, respectively. The structure of the new products was established based on elemental and spectral analysis. Antimicrobial evaluation of some selected examples from the synthesized products was carried out whereby four compounds were found to have moderate activities and one compound showed the highest activity.
\end{abstract}

\section{Introduction}

Sulfonamides have been reported to exhibit antimicrobial [13], antifungal [4], insulin releasing [5], carbonic anhydrase inhibitory [6], anti-inflammatory [7], and antitumor [8], properties. Some active sulfonamides as antibacterial are also known for their immune modifying effects [9]. Also, pyrazole derivatives are known to exhibit diverse bioactivities such as antidepressant [10], anticonvulsant [11], antimicrobial [12], analgesic [13], and antitumor [14] activity and also serve as human acyl-CoA: cholesterol acyltransferase inhibitors [15]. In addition, thiophene compounds are well known to exhibit various biological and medicinal activities such as BACEI inhibitors [16], antitubercular [17], antidepressant [18], antiinflammatory [19], and anti-HIV PR inhibitors [20], and antibreast cancer activities [21]. These facts, coupled with our desire to develop efficacious antimicrobial agents, and in continuation of our work in heterocycles of biological interest [22-25], prompted us to devise an efficient and convenient method of synthesis of hitherto unknown and novel hydrazone, pyridazine, acrylamide, pyrazole, triazolo[1,5a] pyrimidine, thiadiazole, dihydropyrimidine, and thiophene derivatives with a sulfonamide nucleus. Results from assessment of the antimicrobial activity of these newly synthesized compounds are reported in this study.

\section{Experimental Part}

All melting points were determined on an electrothermal Gallenkamp apparatus and are uncorrected. The IR spectra were measured on a Pye-Unicam SP300 instrument in potassium bromide discs. The ${ }^{1} \mathrm{H}$-NMR spectra were recorded on a Varian Mercury VXR-300 spectrometer $(300 \mathrm{MHz})$. The mass spectra were recorded on a GCMS-Q1000-EX Shimadzu and GCMS 5988-A HP spectrometers; the ionizing 
voltage was $70 \mathrm{eV}$. Elemental analyses were carried out by the Microanalytical Center of Cairo University, Giza, Egypt. The biological evaluation of the products $3 a, 3 c, 6,7,8 b, 10,15,16$, and $\mathbf{1 7}$ was carried out at the Medical Mycology Laboratory of the Regional Center for Mycology and Biotechnology of Al-Azhar University, Cairo, Egypt.

3-Oxo-3-phenyl- $N$-(4-sulfamoylphenyl)propionamide (1) and 3-(2-bromoacetyl)-2H-chromen-2-one [26-29] were prepared as previously described.

\subsection{Coupling of 3-Oxo-3-phenyl-N-(4-sulfamoylphenyl)pro- pionamide (1) with the Appropriate Diazonium Salt of Aro- matic Amines}

General Procedure. To a cold solution of 3-oxo-3-phenyl-N(4-sulfamoylphenyl)propionamide (1) $(0.318 \mathrm{~g}, 1 \mathrm{mmol})$ in pyridine $(20 \mathrm{~mL})$, the appropriate diazonium salt of aromatic amine (aniline or 4-methylaniline or 4-chloroaniline or 4methoxy-aniline or 4-nitroaniline) ( $1 \mathrm{mmol}$ ) was added (prepared according to literature procedures) [30]. The addition was carried out portionwise with stirring at $0-5^{\circ} \mathrm{C}$ over a period of $30 \mathrm{~min}$. After complete addition, the reaction mixture was stirred for further $4 \mathrm{hrs}$, then kept in an ice chest for $12 \mathrm{hrs}$, and finally diluted with water. The precipitated solid was collected by filtration, washed with water, dried, and finally recrystallized from the proper solvent to afford the corresponding coupling products $3 \mathbf{a}-\mathbf{e}$.

2.2. 2-[(4-Methoxyphenyl)hydrazono]-3-oxo-3-phenyl-N-(4sulfamoylphenyl)propionamide (3a). Yield (80\%), m.p. $270^{\circ} \mathrm{C}$ (dioxane); IR (KBr) $\nu_{\max }: 3351,3264\left(\mathrm{NH}, \mathrm{NH}_{2}\right), 3073$ (aromatic CH), $1657(\mathrm{C}=\mathrm{O}) \mathrm{cm}^{-1} ;{ }^{1} \mathrm{H}$ NMR $\left(\mathrm{DMSO}_{6}\right): \delta$ $3.75\left(\mathrm{~s}, 3 \mathrm{H}, \mathrm{OCH}_{3}\right), 6.96(\mathrm{~d}, 2 \mathrm{H}, J=9 \mathrm{~Hz}), 7.28(\mathrm{~d}, 2 \mathrm{H}, J=$ $9 \mathrm{~Hz}$ ), 7.30 (s, 2H, $\mathrm{D}_{2} \mathrm{O}$-exchangeable $\left.\mathrm{NH}_{2}\right), 7.61(\mathrm{~d}, 2 \mathrm{H}, J=$ $8 \mathrm{~Hz}$ ), 7.77 (d, 2H, $J=8 \mathrm{~Hz}), 7.79-7.93$ (m, 5H, Ar-H), 11.47 (s, $1 \mathrm{H}, \mathrm{D}_{2} \mathrm{O}$-exchangeable $\left.\mathrm{NH}\right), 13.82\left(\mathrm{~s}, 1 \mathrm{H}, \mathrm{D}_{2} \mathrm{O}\right.$-exchangeable $\mathrm{NH}) ; \mathrm{MS} m / z(\%): 452\left(\mathrm{M}^{+}+2,3.33\right), 452\left(\mathrm{M}^{+}, 18.37\right), 347$ (4.07), 252 (15.66), 196 (3.51), 172 (5.49), 105 (100.0). Anal. Calcd for $\mathrm{C}_{22} \mathrm{H}_{20} \mathrm{~N}_{4} \mathrm{O}_{5} \mathrm{~S}$ (452.49): C, 58.40; H, 4.46; N, 12.38; S, 7.09. Found: C, 58.36; H, 4.36; N, 12.32; S, 7.01\%.

2.3. 2-(p-Tolylhydrazono)-3-oxo-3-phenyl-N-(4-sulfamoylphenyl)propionamide (3b). Yield (75\%), m.p. $280^{\circ} \mathrm{C}$ (dioxane); IR (KBr) $v_{\max }: 3352,3265\left(\mathrm{NH}, \mathrm{NH}_{2}\right), 2924$, 2858 (aliphatic $\mathrm{CH}), 1660(\mathrm{C}=\mathrm{O}) \mathrm{cm}^{-1} ;{ }^{1} \mathrm{H}$ NMR (DMSO$\left.\mathrm{d}_{6}\right): \delta 2.26\left(\mathrm{~s}, 3 \mathrm{H}, \mathrm{CH}_{3}\right), 7.14\left(\mathrm{~s}, 2 \mathrm{H}, \mathrm{D}_{2} \mathrm{O}\right.$-exchangeable $\left.\mathrm{NH}_{2}\right)$, 7.17-7.30 (m, 5H, Ar-H), 7.56 (d, 2H, J = 9 Hz), 7.62 (d, 2H, J $=9 \mathrm{~Hz}), 7.81(\mathrm{~d}, 2 \mathrm{H}, J=8 \mathrm{~Hz}), 7.93(\mathrm{~d}, 2 \mathrm{H}, J=8 \mathrm{~Hz}), 11.37(\mathrm{~s}$, $1 \mathrm{H}, \mathrm{D}_{2} \mathrm{O}$-exchangeable $\left.\mathrm{NH}\right), 13.55$ (s, $1 \mathrm{H}, \mathrm{D}_{2} \mathrm{O}$-exchangeable $\mathrm{NH}) ; \mathrm{MS} m / z$ (\%): $437\left(\mathrm{M}^{+}+1,25.12\right), 436\left(\mathrm{M}^{+}, 35.75\right), 332$ (26.57), 329 (32.85), 279 (51.21), 263 (33.82), 171 (42.03), 157 (31.40), 104 (28.02), 57 (100.0). Anal. Calcd for $\mathrm{C}_{22} \mathrm{H}_{20} \mathrm{~N}_{4} \mathrm{O}_{4} \mathrm{~S}$ (436.49): C, 60.54; H, 4.62; N, 12.84; S, 7.35. Found: C, 60.50; $\mathrm{H}, 4.55 ; \mathrm{N}, 12.72 ; \mathrm{S}, 7.27 \%$.

2.4. 2-(Phenylhydrazono)-3-oxo-3-phenyl-N-(4-sulfamoylphen$y$ l)propionamide $(3 \mathrm{c})$. Yield (70\%), m.p. $282^{\circ} \mathrm{C}$ (dioxane); IR $(\mathrm{KBr}) v_{\max }$ : 3349, $3256\left(\mathrm{NH}, \mathrm{NH}_{2}\right), 3059$ (aromatic $\mathrm{CH}$ ),
$1650(\mathrm{C}=\mathrm{O}) \mathrm{cm}^{-1} ;{ }^{1} \mathrm{H}$ NMR (DMSO-d 6 ): $\delta 7.18\left(\mathrm{~s}, 2 \mathrm{H}, \mathrm{D}_{2} \mathrm{O}-\right.$ exchangeable $\left.\mathrm{NH}_{2}\right), 7.20-7.66(\mathrm{~m}, 5 \mathrm{H}, \mathrm{Ar}-\mathrm{H}), 7.70(\mathrm{~d}, 2 \mathrm{H}, \mathrm{J}=$ $9 \mathrm{~Hz}), 7.76(\mathrm{~d}, 2 \mathrm{H}, J=9 \mathrm{~Hz}), 7.85-8.00(\mathrm{~m}, 5 \mathrm{H}, \mathrm{Ar}-\mathrm{H}), 11.59$ (s, $1 \mathrm{H}, \mathrm{D}_{2} \mathrm{O}$-exchangeable $\left.\mathrm{NH}\right), 13.46\left(\mathrm{~s}, 1 \mathrm{H}, \mathrm{D}_{2} \mathrm{O}\right.$-exchangeable $\mathrm{NH}) ; \mathrm{MS} \mathrm{m} / z$ (\%): $422\left(\mathrm{M}^{+}, 7.6\right), 223$ (1.9), 199 (0.4), 171 (1.8), 121 (20.3), 119 (3.0), 105 (100.0), 77 (57.7). Anal. Calcd for $\mathrm{C}_{21} \mathrm{H}_{18} \mathrm{~N}_{4} \mathrm{O}_{4} \mathrm{~S}$ (422.47): C, 59.71; H, 4.29; N, 13.26; S, 7.59. Found: C, 59.66; H, 4.26; N, 13.30; S, 7.53\%.

2.5. 2-[(4-Chlorophenyl)hydrazono]-3-oxo-3-phenyl-N-(4-sulfamoylphenyl)propionamide (3d). Yield (60\%), m.p. $304^{\circ} \mathrm{C}$ (dioxane); IR (KBr) $v_{\max }: 3364,3262$ and $3152\left(\mathrm{NH}, \mathrm{NH}_{2}\right)$, $1660(\mathrm{C}=\mathrm{O}) \mathrm{cm}^{-1} ;{ }^{1} \mathrm{H}$ NMR (DMSO-d $\left.\mathrm{d}_{6}\right): \delta 7.24\left(\mathrm{~s}, 2 \mathrm{H}, \mathrm{D}_{2} \mathrm{O}-\right.$ exchangeable $\left.\mathrm{NH}_{2}\right), 7.52(\mathrm{~d}, 2 \mathrm{H}, J=8 \mathrm{~Hz}), 7.62(\mathrm{~d}, 2 \mathrm{H}, J=$ $8 \mathrm{~Hz}), 7.64(\mathrm{~d}, 2 \mathrm{H}, J=9 \mathrm{~Hz}), 7.83(\mathrm{~d}, 2 \mathrm{H}, J=9 \mathrm{~Hz}), 7.85-7.93$ (m, 5H, Ar-H), 11.21 (s, 1H, D $2 \mathrm{O}$-exchangeable $\mathrm{NH}), 13.05$ (s, $1 \mathrm{H}, \mathrm{D}_{2} \mathrm{O}$-exchangeable $\left.\mathrm{NH}\right) ; \mathrm{MS} m / z(\%)$ : $457\left(\mathrm{M}^{+}+1\right.$, 13.25), 456 ( $\left.\mathrm{M}^{+}, 16.64\right), 329$ (8.10), 285 (11.19), 199 (10.90), 128 (11.49), 105 (100.0). Anal. Calcd for $\mathrm{C}_{21} \mathrm{H}_{17} \mathrm{ClN}_{4} \mathrm{O}_{4} \mathrm{~S}$ (456.91): C, 55.20; H, 3.75; Cl, 7.76; N, 12.26; S, 7.02. Found: C, 55.15; H, $3.66 ; \mathrm{Cl}, 7.66 ; \mathrm{N}, 12.21 ; \mathrm{S}, 7.00 \%$.

2.6. 2-[(4-Nitrophenyl)hydrazono]-3-oxo-3-phenyl-N-(4-sulfamoylphenyl)propionamide (3e). Yield (55\%), m.p. $302^{\circ} \mathrm{C}$ (dioxane); IR (KBr) $v_{\max }: 3368,3265$ and $3161\left(\mathrm{NH}, \mathrm{NH}_{2}\right)$, $1664(\mathrm{C}=\mathrm{O}) \mathrm{cm}^{-1} ;{ }^{1} \mathrm{H}$ NMR (DMSO-d $\left.\mathrm{d}_{6}\right): \delta 7.26\left(\mathrm{~s}, 2 \mathrm{H}, \mathrm{D}_{2} \mathrm{O}-\right.$ exchangeable $\left.\mathrm{NH}_{2}\right), 7.59(\mathrm{~d}, 2 \mathrm{H}, J=9 \mathrm{~Hz}), 7.70(\mathrm{~d}, 2 \mathrm{H}, J=$ $9 \mathrm{~Hz}$ ), 7.78-7.89 (m, 5H, Ar-H), 7.91 (d, 2H, J = 8 Hz), 8.20 (d, $2 \mathrm{H}, J=8 \mathrm{~Hz}$ ), 11.08 (s, $1 \mathrm{H}, \mathrm{D}_{2} \mathrm{O}$-exchangeable $\left.\mathrm{NH}\right), 12.45$ (s, $1 \mathrm{H}, \mathrm{D}_{2} \mathrm{O}$-exchangeable $\left.\mathrm{NH}\right)$; $\mathrm{MS} m / z(\%): 468\left(\mathrm{M}^{+}+1,3.89\right)$, $467\left(\mathrm{M}^{+}, 7.25\right), 362$ (4.71), 296 (4.26), 199 (4.33), 172 (19.88), 105 (100.0). Anal. Calcd for $\mathrm{C}_{21} \mathrm{H}_{17} \mathrm{~N}_{5} \mathrm{O}_{6} \mathrm{~S}$ (467.46): C, 53.96; H, 3.67; N, 14.98; S, 6.86. Found: C, 53.87; H, 3.59; N, 14.88; S, $6.78 \%$.

2.7. N-[4-(Aminosulfonyl)phenyl]-5-cyano-6-imino-4-phenylp-tolyl-1,6-dihydropyridazine-3-carboxamide (4). To a solution of (3b) $(0.436 \mathrm{~g}, 1 \mathrm{mmol})$ and malononitrile $(1 \mathrm{mmol})$ in dioxane $(20 \mathrm{~mL})$, few drops of piperidine were added and the reaction mixture was refluxed for $6 \mathrm{hrs}$. The solid product that formed was filtered off, washed with ethanol, and then recrystallized from the proper solvent to give 4 .

Yield (55\%), m.p. $>300^{\circ} \mathrm{C}(\mathrm{DMF})$; IR (KBr) $\nu_{\max }: 3462$, 3305 and $3181\left(\mathrm{NH}, \mathrm{NH}_{2}\right), 2203(\mathrm{C} \equiv \mathrm{N}), 1637(\mathrm{C}=\mathrm{O}) \mathrm{cm}^{-1}$; ${ }^{1} \mathrm{H}$ NMR (DMSO-d $\left.)_{6}\right): \delta 2.49\left(\mathrm{~s}, 3 \mathrm{H}, \mathrm{CH}_{3}\right), 7.32(\mathrm{~d}, 2 \mathrm{H}$ $\mathrm{D}_{2} \mathrm{O}$-exchangeable $\left.\mathrm{NH}_{2}\right), 7.40(\mathrm{~d}, 2 \mathrm{H}, J=8 \mathrm{~Hz}), 7.59(\mathrm{~d}$, $2 \mathrm{H}, J=9 \mathrm{~Hz}), 7.67-7.90(\mathrm{~m}, 9 \mathrm{H}, \mathrm{Ar}-\mathrm{H}), 11.20\left(\mathrm{~s}, 1 \mathrm{H}, \mathrm{D}_{2} \mathrm{O}-\right.$ exchangeable $\mathrm{NH}), 14.60$ ( $\mathrm{s}, 1 \mathrm{H}, \mathrm{D}_{2} \mathrm{O}$-exchangeable $\left.\mathrm{NH}\right) ; \mathrm{MS}$ $m / z$ (\%): $484\left(\mathrm{M}^{+}, 2.32\right), 409$ (7.42), 393 (9.09), 313 (6.94), 285 (6.58), 199 (7.89), 91 (16.51), 77 (26.56), 69 (100.0). Anal. Calcd for $\mathrm{C}_{25} \mathrm{H}_{20} \mathrm{~N}_{6} \mathrm{O}_{3} \mathrm{~S}$ (484.54): C, 61.97; $\mathrm{H}, 4.16 ; \mathrm{N}, 17.34 ; \mathrm{S}, 6.62$. Found: C, 61.92; H, 4.11; N, 17.30; S, 6.59\%.

2.8. 4-(6-Benzoyl-3-ethoxy-5-oxo-2-p-tolyl-2,3-dihydro-1,2,4triazin-4-(5H)-yl)benzenesulfonamide (5). To a solution of the compound $\mathbf{3 b}(0.436 \mathrm{~g}, 1 \mathrm{mmol})$ in acetic acid $(20 \mathrm{~mL})$, triethyl orthoformate $(0.2 \mathrm{~mL}, 1 \mathrm{mmol})$ was added and the reaction mixture was refluxed for $8 \mathrm{hrs}$; then it was left to cool. 
So the solid product formed was filtered off, washed with $\mathrm{EtOH}$, and dried. Recrystallization from dioxane afforded 4-(6-benzoyl-3-ethoxy-5-oxo-2-p-tolyl-2,3-dihydro-1,2,4triazin-4-(5H)-yl)benzenesulfonamide (5).

Yield (50\%), m.p. $296^{\circ} \mathrm{C}$ (DMF); IR (KBr) $\nu_{\max }: 3370,3311$ and $3236\left(\mathrm{NH}, \mathrm{NH}_{2}\right), 3056$ (aromatic $\mathrm{CH}$ ), 2982 (aliphatic$\mathrm{H}), 1678(\mathrm{C}=\mathrm{O}) \mathrm{cm}^{-1} ;{ }^{1} \mathrm{H}$ NMR $\left(\mathrm{DMSO}_{-} \mathrm{d}_{6}\right): \delta 1.32(\mathrm{t}, 3 \mathrm{H}, J=$ $7.2 \mathrm{~Hz}, \mathrm{CH}_{3}$ ), $2.24\left(\mathrm{~s}, 3 \mathrm{H}, \mathrm{CH}_{3}\right), 4.27\left(\mathrm{q}, 2 \mathrm{H}, J=7.2, \mathrm{CH}_{2}\right), 5.94$ $(\mathrm{S}, 1 \mathrm{H}), 7.36\left(\mathrm{~s}, 2 \mathrm{H}, \mathrm{D}_{2} \mathrm{O}\right.$-exchangeable $\left.\mathrm{NH}_{2}\right), 7.37-7.57(\mathrm{~m}$, 9H, Ar-H), 7.60 (d, 2H, $J=9 \mathrm{~Hz}), 7.71(\mathrm{~d}, 2 \mathrm{H}, J=9 \mathrm{~Hz})$; MS $m / z$ (\%): $494\left(\mathrm{M}^{+}+2,0.1\right), 492\left(\mathrm{M}^{+}, 0.5\right), 352(0.42), 335$ (1.6), 156 (0.6), 105 (100.0), 91 (1.5). Anal. Calcd for $\mathrm{C}_{25} \mathrm{H}_{24} \mathrm{~N}_{4} \mathrm{O}_{5} \mathrm{~S}$ (492.56): C, 60.96; H, 4.91; N, 11.37; S, 6.51. Found: C, 60.88; $\mathrm{H}, 4.85 ; \mathrm{N}, 11.31 ; \mathrm{S}, 6.50 \%$.

2.9. 2-Benzoyl-3-dimethylamino-N-(4-sulfamoylphenyl)acrylamide (6). A mixture of the compound 1 (3.18 g, $10 \mathrm{mmol})$ and $N, N$-dimethylformamide dimethyl acetal (DMF-DMA) $(10 \mathrm{mmol})$ in dry dioxane $(30 \mathrm{~mL})$ was refluxed for $6 \mathrm{hrs}$; then it was left to cool at room temperature. The yellow precipitated product was filtered off, washed with petroleum ether, and dried. Crystallization from $\mathrm{MeOH}$ was carried out to give 2-benzoyl-3-dimethylamino- $N$-(4sulfamoylphenyl)acryl-amide (6) in $44 \%$ yield.

Yield (44\%), m.p. $190^{\circ} \mathrm{C}(\mathrm{MeOH})$; IR (KBr) $v_{\max }$ : 3250, $3112\left(\mathrm{NH}, \mathrm{NH}_{2}\right), 3056$ (aromatic $\left.\mathrm{CH}\right), 1683(\mathrm{C}=\mathrm{O}), \mathrm{cm}^{-1}$; ${ }^{1} \mathrm{H}$ NMR (DMSO-d 6$): \delta 3.42\left(\mathrm{~s}, 6 \mathrm{H}, 2 \mathrm{CH}_{3}\right), 6.62(\mathrm{~d}, 2 \mathrm{H}, J$ $=9 \mathrm{~Hz}), 7.03\left(\mathrm{~s}, 2 \mathrm{H}, \mathrm{D}_{2} \mathrm{O}\right.$-exchangeable $\left.\mathrm{NH}_{2}\right), 7.28-7.31(\mathrm{~m}$, $5 \mathrm{H}, \operatorname{Ar}-\mathrm{H}), 7.58$ (d, $2 \mathrm{H}, J=9 \mathrm{~Hz}), 8.10$ (s, $1 \mathrm{H}$, olefinic- $\mathrm{H})$, 9.98 (s, $1 \mathrm{H}, \mathrm{D}_{2} \mathrm{O}$-exchangeable $\left.\mathrm{NH}\right) ; \mathrm{MS} m / z(\%): 373\left(\mathrm{M}^{+}\right.$, 0.4), 291 (1.1), 190 (0.5), 155 (16.3), 105 (100.0). Anal. Calcd for $\mathrm{C}_{18} \mathrm{H}_{19} \mathrm{~N}_{3} \mathrm{O}_{4} \mathrm{~S}$ (373.43): C, 57.90; H, 5.13; N, 11.25; S, 8.59. Found: C, 57.89; H, 5.03; N, 11.15; S, 8.61\%.

2.10. 5-Phenyl-[1,2,4]triazolo[1,5-va]pyrimidine-6-carboxylic Acid (4-sulfamoylphenyl)amide (7). To a solution of the compound $6(0.37 \mathrm{~g}, 1 \mathrm{mmol})$ in acetic acid $(20 \mathrm{~mL})$, amino triazolo $(0.1 \mathrm{~g}, 1 \mathrm{mmol})$ was added and the reaction mixture was refluxed for $8 \mathrm{hrs}$; then it was left to cool. The solid product formed was filtered off, washed with EtOH, and dried. Recrystallization from dioxane afforded 5-phenyl-[1,2,4]triazolo[1,5-a]-pyrimidine-6-carboxylic acid(4-sul-famoylphenyl)amide (7).

Yield (48\%), m.p. $290^{\circ} \mathrm{C}$ (dioxane); IR (KBr) $\nu_{\max }: 3261$, $3110\left(\mathrm{NH}, \mathrm{NH}_{2}\right), 1685(\mathrm{C}=\mathrm{O}), \mathrm{cm}^{-1} ;{ }^{1} \mathrm{H}$ NMR $\left(\mathrm{DMSO}-\mathrm{d}_{6}\right): \delta$ $6.61\left(\mathrm{~s}, 1 \mathrm{H}, \mathrm{D}_{2} \mathrm{O}\right.$-exchangeable $\left.\mathrm{NH}_{2}\right), 7.05(\mathrm{~d}, 2 \mathrm{H}, J=9 \mathrm{~Hz})$, $7.34-7.44$ (m, 5H, Ar-H), 7.50 (d, 2H, J = 9 Hz), 7.68 (s, 1H, triazole-H), 8.09 (s, $1 \mathrm{H}$, pyrimidine- $\mathrm{H}), 8.48\left(\mathrm{~s}, 1 \mathrm{H}, \mathrm{D}_{2} \mathrm{O}-\right.$ exchangeable $\mathrm{NH})$; $\mathrm{MS} m / z(\%): 397\left(\mathrm{M}^{+}+3,20.0\right), 394(\mathrm{M}+$, 0.2), 157 (16.0), 121 (52.0), 76 (84.0), 63 (100.0). Anal. Calcd for $\mathrm{C}_{18} \mathrm{H}_{14} \mathrm{~N}_{6} \mathrm{O}_{3} \mathrm{~S}$ (394.41): C, 54.82; $\mathrm{H}, 3.58 ; \mathrm{N}, 21.31 ; \mathrm{S}, 8.13$. Found: C, 54.80; H, 3.53; N, 21.25; S, 8.01\%.

\subsection{2-Benzoyl-3-aryl-2-yl-N-(4-sulfamoylphenyl)acrylamide $(8 a, b)$}

General Procedure. To a solution of (1) (0.318 g, 1 mmol) and the appropriate aromatic aldehydes $(1 \mathrm{mmol})$ in dioxane
$(20 \mathrm{~mL})$, few drops of piperidine were added and the reaction mixture was refluxed for $6 \mathrm{hrs}$. So the solid product formed was filtered off, washed with $\mathrm{EtOH}$, dried, and finally recrystallized from the proper solvent to give $\mathbf{8 a}, \mathbf{b}$.

2.12. 2-Benzoyl-3-furan-2-yl-N-(4-sulfamoylphenyl)acrylamide $(\mathbf{8 a})$. Yield (45\%), m.p. $220^{\circ} \mathrm{C}(\mathrm{EtOH})$; IR $(\mathrm{KBr}) \nu_{\max }$ : 3349, $3255\left(\mathrm{NH}, \mathrm{NH}_{2}\right), 1687(\mathrm{C}=\mathrm{O}) \mathrm{cm}^{-1} ;{ }^{1} \mathrm{H}$ NMR (DMSO$\left.\mathrm{d}_{6}\right): \delta 7.43\left(\mathrm{~s}, 2 \mathrm{H}, \mathrm{D}_{2} \mathrm{O}\right.$-exchangeable $\left.\mathrm{NH}_{2}\right), 7.46-7.61(\mathrm{~m}, 8 \mathrm{H}$, $\operatorname{Ar}-\mathrm{H}), 7.64(\mathrm{~d}, 2 \mathrm{H}, J=9 \mathrm{~Hz}), 7.94(\mathrm{~d}, 2 \mathrm{H}, J=9 \mathrm{~Hz}), 8.48$ (s, $1 \mathrm{H}$, olefinic-H), 9.14 (s, $1 \mathrm{H}, \mathrm{D}_{2} \mathrm{O}$-exchangeable $\mathrm{NH}$ ); $\mathrm{MS} \mathrm{m} / z$ (\%): $396\left(\mathrm{M}^{+}, 0.1\right), 225$ (0.3), 197 (0.4), 171 (22.4), 157 (2.8), 105 (100.0). Anal. Calcd for $\mathrm{C}_{20} \mathrm{H}_{16} \mathrm{~N}_{2} \mathrm{O}_{5} \mathrm{~S}$ (396.42): C, 60.60; $\mathrm{H}$, 4.07; N, 7.07; S, 8.09. Found: C, 60.53; H, 4.01; N, 7.11; S, 8.00\%.

2.13. 2-Benzoyl-3-(1,3-diphenyl-1H-pyrazol-4-yl)-N-(4-sulfamoylphenyl)acrylamide (8b). Yield (50\%), m.p. $180^{\circ} \mathrm{C}$ $(\mathrm{EtOH}) ; \mathrm{IR}(\mathrm{KBr}) v_{\max }: 3341,3261\left(\mathrm{NH}, \mathrm{NH}_{2}\right), 3060$ (aromatic CH), $1675(\mathrm{C}=\mathrm{O}) \mathrm{cm}^{-1} ;{ }^{1} \mathrm{H}$ NMR (DMSO-d 6 ): $\delta 7.25\left(\mathrm{~s}, 2 \mathrm{H}, \mathrm{D}_{2} \mathrm{O}\right.$-exchangeable $\left.\mathrm{NH}_{2}\right), 7.49-7.00(\mathrm{~m}, 15 \mathrm{H}$, Ar-H), 7.64 (s, 1H, pyrazole-H), 7.77 (d, 2H, J = 9Hz), $7.81(\mathrm{~d}, 2 \mathrm{H}, J=9 \mathrm{~Hz}), 7.86$ (s, $1 \mathrm{H}$, olefinic-H), 10.85 (s, $1 \mathrm{H}$, $\mathrm{D}_{2} \mathrm{O}$-exchangeable $\left.\mathrm{NH}\right)$; MS $m / z(\%): 548\left(\mathrm{M}^{+}, 0.1\right), 217$ (16.7), 199 (20.0), 172 (16.7), 105 (96.7), 92 (20.0), 77 (100.0). Anal. Calcd for $\mathrm{C}_{31} \mathrm{H}_{24} \mathrm{~N}_{4} \mathrm{O}_{4} \mathrm{~S}$ (548.63): C, 67.87; $\mathrm{H}, 4.41 ; \mathrm{N}$, 10.21; S, 5.84. Found: C, 67.82; H, 4.35; N, 10.16; S, 5.78\%.

2.14. 5-Furan-2-yl-3-phenyl-2,3-dihydro-1-H-pyrazole-4-carboxylic Acid (4-sulfamoylphenyl)amide (9a). To a solution of the compound $8 \mathbf{a}(0.4 \mathrm{~g}, 1 \mathrm{mmol})$ in dioxane $(20 \mathrm{~mL})$, hydrazine hydrate $(80 \%, 0.2 \mathrm{~mL}, 1 \mathrm{mmol})$ was added and the reaction mixture was refluxed for $6 \mathrm{hrs}$; then it was left to cool. So the solid product formed was filtered off, washed with $\mathrm{EtOH}$, and dried. Recrystallization from dioxane afforded 9a.

Yield (40\%), m.p. $244^{\circ} \mathrm{C}(\mathrm{EtOH})$; IR (KBr) $\nu_{\max }: 3281,3111$ $\left(\mathrm{NH}, \mathrm{NH}_{2}\right), 1666(\mathrm{C}=\mathrm{O}) \mathrm{cm}^{-1} ;{ }^{1} \mathrm{H}$ NMR $\left(\mathrm{DMSO}_{-} \mathrm{d}_{6}\right): \delta 6.33-$ $6.37(\mathrm{~m}, 2 \mathrm{H}), 7.21\left(\mathrm{~s}, 2 \mathrm{H}, \mathrm{D}_{2} \mathrm{O}\right.$-exchangeable $\left.\mathrm{NH}_{2}\right), 7.44(\mathrm{~d}$, $2 \mathrm{H}, J=9 \mathrm{~Hz}), 7.50(\mathrm{~d}, 2 \mathrm{H}, J=9 \mathrm{~Hz}), 7.56-7.75(\mathrm{~m}, 6 \mathrm{H}, \mathrm{Ar}-$ $\mathrm{H}), 9.99$ (s, $1 \mathrm{H}, \mathrm{D}_{2} \mathrm{O}$-exchangeable $\left.\mathrm{NH}\right), 10.42$ (s, $1 \mathrm{H}, \mathrm{D}_{2} \mathrm{O}$ exchangeable $\mathrm{NH})$; MS $m / z(\%): 408\left(\mathrm{M}^{+}, 3.5\right), 171$ (20.4), 142 (3.5), 77 (100). Anal. Calcd for $\mathrm{C}_{20} \mathrm{H}_{16} \mathrm{~N}_{4} \mathrm{O}_{4} \mathrm{~S}$ (408.44): C, 58.81; H, 3.95; N, 13.72; S, 7.85. Found: C, 58.79; H, 3.93; N, 13.65; S, 7.80\%.

2.15. 2-Benzoyl-3-ethoxy-N-(4-sulfamoylphenyl)acrylamide (10). To a solution of the compound 1 (3.18 g, $10 \mathrm{mmol})$ in acetic anhydride $(20 \mathrm{~mL})$, triethyl orthoformate $(2 \mathrm{~mL}$, $10 \mathrm{mmol}$ ) was added and the reaction mixture was refluxed for $8 \mathrm{hrs}$; then it was left to cool. So the solid product formed was filtered off, washed with $\mathrm{EtOH}$, and dried. Recrystallization from dioxane afforded 2-benzoyl-3-ethoxy$N$-(4-sulfamoylphenyl)acrylamide (10).

Yield (60\%), m.p. $280^{\circ} \mathrm{C}(\mathrm{DMF}) ; \mathrm{IR}(\mathrm{KBr}) \nu_{\max }: 3359,3239$ and $3107\left(\mathrm{NH}, \mathrm{NH}_{2}\right), 1655(\mathrm{C}=\mathrm{O}) \mathrm{cm}^{-1} ;{ }^{1} \mathrm{H}$ NMR (DMSO$\left.\mathrm{d}_{6}\right): \delta 1.26\left(\mathrm{t}, 3 \mathrm{H}, J=7.2 \mathrm{~Hz}, \mathrm{CH}_{3}\right), 4.32\left(\mathrm{q}, 2 \mathrm{H}, J=7.2, \mathrm{CH}_{2}\right)$, 7.16 (s, 2H, $\mathrm{D}_{2} \mathrm{O}$-exchangeable $\left.\mathrm{NH}_{2}\right), 7.40(\mathrm{~d}, 2 \mathrm{H}, J=9 \mathrm{~Hz})$, 7.69 (d, 2H, J = 9 Hz), 7.87-8.00 (m, 5H, Ar-H), 8.63 (s, 1H, $\mathrm{CH}), 11.25$ (s, $1 \mathrm{H}, \mathrm{D}_{2} \mathrm{O}$-exchangeable $\mathrm{NH}$ ); $\mathrm{MS} m / z$ (\%): 374 
$\left(\mathrm{M}^{+}, 0.5\right), 370(0.6), 301(72.7), 198(18.0), 172(33.6), 156$ (59.6), 105 (70.8), 77 (85.0), 65 (100.0). Anal. Calcd for $\mathrm{C}_{18} \mathrm{H}_{18} \mathrm{~N}_{2} \mathrm{O}_{5} \mathrm{~S}$ (374.42): C, 57.74; H, 4.85; N, 7.48; S, 8.56. Found: C, 57.70; H, 4.76 ; N, 7.41; S, 8.50\%.

2.16. 4-(6-Phenyl-2-thioxo-2,3-dihydropyrimidin-4-ylamino) benzenesulfonamide (11). To a mixture of $\mathbf{1}(0.318 \mathrm{~g}, 1 \mathrm{mmol})$ and thiourea $(0.076 \mathrm{~g}, 1 \mathrm{mmol})$ in dimethylformamide $(20 \mathrm{~mL})$, triethylamine $(0.5 \mathrm{~mL})$ was added and the reaction mixture was refluxed for $6 \mathrm{hrs}$; then it was left to cool. The precipitated product was filtered off and purified by recrystallization from the suitable solvent to afford the corresponding $\mathbf{1 1}$.

Yield (45\%), m.p. $>300^{\circ} \mathrm{C}$ (dioxane); IR (KBr) $\nu_{\max }$ : 3500-3368 (NH, $\left.\mathrm{NH}_{2}\right), 3057$ (aromatic $\left.\mathrm{CH}\right), 1675(\mathrm{C}=\mathrm{O})$ $\mathrm{cm}^{-1} ;{ }^{1} \mathrm{H}$ NMR (DMSO-d $\left.\mathrm{D}_{6}\right): \delta 7.27$ (s, 2H, $\mathrm{D}_{2} \mathrm{O}$-exchangeable $\left.\mathrm{NH}_{2}\right), 7.54(\mathrm{~d}, 2 \mathrm{H}, J=9 \mathrm{~Hz}), 7.66(\mathrm{~d}, 2 \mathrm{H}, J=9 \mathrm{~Hz}), 7.68-7.97$ (m, 6H, Ar-H), 11.85 (s, $1 \mathrm{H}, \mathrm{D}_{2} \mathrm{O}$-exchangeable $\mathrm{NH}$ ), 12.23 (s, $1 \mathrm{H}, \mathrm{D}_{2} \mathrm{O}$-exchangeable $\left.\mathrm{NH}\right)$. Anal. Calcd for $\mathrm{C}_{16} \mathrm{H}_{14} \mathrm{~N}_{4} \mathrm{O}_{2} \mathrm{~S}_{2}$ (358.44): C, 53.61; H, 3.94; N, 15.63; S, 17.89. Found: C, 53. 60; H, 3.90; N, 15.59; S, 17.84.\%.

2.17. 2-Benzoyl-3-mercapto-3-phenylamino-N-(4-sulfamoylphenyl)acrylamide (13). To a stirred solution of potassium hydroxide $(0.11 \mathrm{~g}, 1 \mathrm{mmol})$ in dimethylformamide $(20 \mathrm{~mL})$ the $1(0.318 \mathrm{~g}, 1 \mathrm{mmol})$ was added. After stirring for $30 \mathrm{~min}$, phenylisothiocyanate $(0.27 \mathrm{~g}, 0.24 \mathrm{~mL}, 1 \mathrm{mmol})$ was added to the resulting mixture and stirring was continued for $6 \mathrm{~h}$; then it was poured over crushed ice containing hydrochloric acid. The solid product formed was filtered off, washed with water, dried, and finally recrystallized from dioxane to afford $\mathbf{1 3 .}$

Yield (40\%), m.p. $230^{\circ} \mathrm{C}$ (dioxane); IR (KBr) $\nu_{\max }: 3350$, $3256\left(\mathrm{NH}, \mathrm{NH}_{2}\right), 1689(\mathrm{C}=\mathrm{O}), 1661(\mathrm{C}=\mathrm{O}), \mathrm{cm}^{-1} ;{ }^{1} \mathrm{H}$ NMR $\left(\mathrm{DMSO}_{6} \mathrm{~d}_{6}\right): \delta 7.19\left(\mathrm{~s}, 2 \mathrm{H}, \mathrm{D}_{2} \mathrm{O}\right.$-exchangeable $\left.\mathrm{NH}_{2}\right), 7.39(\mathrm{~d}$, $2 \mathrm{H}, J=9 \mathrm{~Hz}), 7.69(\mathrm{~d}, 2 \mathrm{H}, J=9 \mathrm{~Hz}), 7.71-7.90(\mathrm{~m}, 10 \mathrm{H}$, Ar-H), 10.39 (s, 1H, D $\mathrm{D}_{2} \mathrm{O}$-exchangeable $\mathrm{NH}$ ), 11.13 (s, $1 \mathrm{H}$, $\mathrm{D}_{2} \mathrm{O}$-exchang-eable $\left.\mathrm{NH}\right), 12.76$ (s, 1H, SH); MS $m / z$ (\%): 455 $\left(\mathrm{M}^{+}+2,26.3\right), 453\left(\mathrm{M}^{+}, 1.5\right), 121$ (26.3), 105 (100.0), 91 (47.4), 89 (36.8), 77 (89.5). Anal. Calcd for $\mathrm{C}_{22} \mathrm{H}_{19} \mathrm{~N}_{3} \mathrm{O}_{4} \mathrm{~S}_{2}$ (453.54): C, 58.26; H, 4.22; N, 9.26; S, 14.14. Found: C, 58.22; H, 4.20; N, 9.20; S, 14.11\%.

2.18. N-[4-(Aminosulfonyl)phenyl]-2-anilino-4-phenyl-thiophene-5-benzoyl-3-carboxamide (14). To a mixture of acrylamide 13 (1 mmol) and 2-bromo-1-phenylethanone $(0.2 \mathrm{~g}, 1 \mathrm{mmol})$ in dimethylformamide $(20 \mathrm{~mL})$, triethylamine $(0.5 \mathrm{~mL})$ was added and the reaction mixture was refluxed for $6 \mathrm{hrs}$; then it was left to cool. The precipitated product was filtered off and purified by recrystallization from dioxane to afford the corresponding $N$-[4-(aminosulfonyl)phenyl]2-anilino-4-phenyl-thiophene-5-benzoyl-3-carboxamide (14).

Yield (40\%), m.p. $280^{\circ} \mathrm{C}$ (dioxane); IR (KBr) $v_{\max }: 3425$, 3276, $3111\left(\mathrm{NH}, \mathrm{NH}_{2}\right.$ ), 3059 (aromatic $\left.\mathrm{CH}\right), 1698(\mathrm{C}=\mathrm{O}), 1658$ $(\mathrm{C}=\mathrm{O}), \mathrm{cm}^{-1} ;{ }^{1} \mathrm{H}$ NMR (DMSO-d 6$): \delta 7.19\left(\mathrm{~s}, 2 \mathrm{H}, \mathrm{D}_{2} \mathrm{O}-\right.$ exchangeable $\left.\mathrm{NH}_{2}\right), 7.27-7.73(\mathrm{~m}, 15 \mathrm{H}, \mathrm{Ar}-\mathrm{H}), 7.76(\mathrm{~d}, 2 \mathrm{H}, \mathrm{J}=$ $9 \mathrm{~Hz}), 8.22(\mathrm{~d}, 2 \mathrm{H}, J=9 \mathrm{~Hz}), 9.55\left(\mathrm{~s}, 1 \mathrm{H}, \mathrm{D}_{2} \mathrm{O}\right.$-exchangeable $\mathrm{NH}$ ), 9.98 (s, $1 \mathrm{H}, \mathrm{D}_{2} \mathrm{O}$-exchangeable $\mathrm{NH}$ ); $\mathrm{MS} m / z$ (\%): 555
$\left(\mathrm{M}^{+}+2,16.0\right), 553\left(\mathrm{M}^{+}, 1.5\right), 450$ (20.0), $395(20.0), 198(24), 171$ (36.0), 156 (24.0), 105 (92.0), 91 (16.0), 76 (20.0). Anal. Calcd for $\mathrm{C}_{30} \mathrm{H}_{23} \mathrm{~N}_{3} \mathrm{O}_{4} \mathrm{~S}_{2}$ (553.66): C, 65.08; H, 4.19; N, 7.59; S, 11.58. Found: C, 65.02; H, 4.15; N, 7.55; S, 11.54\%.

2.19. Synthesis of $15,16,17,18$, and 20. To a stirred solution of potassium hydroxide $(0.11 \mathrm{~g}, 1 \mathrm{mmol})$ in DMF $(20 \mathrm{~mL})$, compound $1(0.318 \mathrm{~g}, 1 \mathrm{mmol})$ was added. After stirring for $30 \mathrm{~min}$, phenyl isothiocyanate $(0.27 \mathrm{~g}, 1 \mathrm{mmol})$ was added to the resulting mixture. Stirring was continued for $6 \mathrm{hrs}$, and then chloroacetone, ethyl chloroacetate, 3-(2bromoacetyl)-2H-chromen-2-one, methyl iodide, or 2-oxo$N$-phenylpropane hydrazonoyl chloride $(1 \mathrm{mmol})$ was added portionwise over a period of $30 \mathrm{~min}$. After the addition was complete, the reaction mixture was stirred for additional $12 \mathrm{~h}$, during which the reactant dissolved and a yellow product precipitated. The solid product was filtered off, washed with $\mathrm{EtOH}$, and dried; recrystallization from proper solvent afforded 15, 16, 17, 18, and 20.

2.20. N-[4-(Aminosulfonyl)phenyl]-2-anilino-4-phenyl-thiophene-5-acetyl-3-carboxamide (15). Yield (65\%), m.p. $268^{\circ} \mathrm{C}$ (dioxane); IR (KBr) $\nu_{\max }: 3371,3289$ and $3220\left(\mathrm{NH}, \mathrm{NH}_{2}\right)$, 3059 (aromatic $\mathrm{CH}$ ), $1636(\mathrm{C}=\mathrm{O}), \mathrm{cm}^{-1}$; ${ }^{1} \mathrm{H}$ NMR (DMSO$\left.\mathrm{d}_{6}\right): \delta 1.72\left(\mathrm{~s}, 3 \mathrm{H}, \mathrm{CH}_{3}\right), 7.11\left(\mathrm{~s}, 2 \mathrm{H}, \mathrm{D}_{2} \mathrm{O}\right.$-exchangeable $\left.\mathrm{NH}_{2}\right)$, $7.13(\mathrm{~d}, 2 \mathrm{H}, J=9 \mathrm{~Hz}), 7.38-7.46(\mathrm{~m}, 10 \mathrm{H}, \operatorname{Ar}-\mathrm{H}), 7.63(\mathrm{~d}$, $2 \mathrm{H}, J=9 \mathrm{~Hz}), 9.46\left(\mathrm{~s}, 1 \mathrm{H}, \mathrm{D}_{2} \mathrm{O}\right.$-exchangeable $\left.\mathrm{NH}\right), 9.88$ (s, $1 \mathrm{H}, \mathrm{D}_{2} \mathrm{O}$-exchangeable $\left.\mathrm{NH}\right) ;{ }^{13} \mathrm{C}$ NMR (DMSO- $\mathrm{d}_{6}$ ): $\delta$ $28.15,118.68,119.93,123.88,126.36,127.85,128.42,128.71$, $129.20,129.45,134.89,134.97,138.46,140.91,141.32,145.33$, 157.02, 162.59, 189.30; MS $m / z(\%): 492\left(\mathrm{M}^{+}+1,13.2\right), 491$ $\left(\mathrm{M}^{+}, 15.1\right), 320$ (28.3), 292 (11.3), 187 (20.8), 105 (100.0), 77 (94.3). Anal. Calcd for $\mathrm{C}_{25} \mathrm{H}_{21} \mathrm{~N}_{3} \mathrm{O}_{4} \mathrm{~S}_{2}$ (491.59): C, 61.08; $\mathrm{H}$, 4.31; N, 8.55; S, 13.04. Found: C, 61.00; H, 4.24; N, 8.5; S, $13.11 \%$.

2.21. 3-Phenyl-5-phenylamino-4-(4-sulfamoylphenyl-carbamoyl)thiophene-2-carboxylic Acid Ethyl Ester (16). Yield (55\%), m.p. $280^{\circ} \mathrm{C}$ (dioxane); IR (KBr) $\nu_{\max }: 3367,3310$ and $3235(\mathrm{NH}$, $\mathrm{NH}_{2}$ ), 3060 (aromatic $\left.\mathrm{CH}\right), 1713(\mathrm{C}=\mathrm{O}), 1635(\mathrm{C}=\mathrm{O}) \mathrm{cm}^{-1}$; ${ }^{1} \mathrm{H} \mathrm{NMR}\left(\mathrm{DMSO}-\mathrm{d}_{6}\right): \delta 1.01\left(\mathrm{~s}, 3 \mathrm{H}, J=7.2 \mathrm{~Hz}, \mathrm{CH}_{3}\right), 4.04(\mathrm{q}$, $\left.2 \mathrm{H}, J=7.2 \mathrm{~Hz}, \mathrm{CH}_{2}\right), 7.08-7.13(\mathrm{~m}, 5 \mathrm{H}, \mathrm{Ar}-\mathrm{H}), 7.21(\mathrm{~d}, 2 \mathrm{H}, J$ $=9 \mathrm{~Hz}), 7.39-7.44\left(\mathrm{~m}, 7 \mathrm{H}, \mathrm{Ar}-\mathrm{H}\right.$ and $\left.\mathrm{NH}_{2}\right), 7.63(\mathrm{~d}, 2 \mathrm{H}, J=$ $9 \mathrm{~Hz}$ ), 9.54 (s, $1 \mathrm{H}, \mathrm{D}_{2} \mathrm{O}$-exchangeable $\left.\mathrm{NH}\right), 9.73\left(\mathrm{~s}, 1 \mathrm{H}, \mathrm{D}_{2} \mathrm{O}\right.$ exchangeable NH); MS m/z (\%): $521\left(\mathrm{M}^{+}, 26.3\right), 322$ (15.8), 218 (100.0), 199 (15.8), 77 (86.0). Anal. Calcd for $\mathrm{C}_{26} \mathrm{H}_{23} \mathrm{~N}_{3} \mathrm{O}_{5} \mathrm{~S}_{2}$ (521.62): C, 59.87; H, 4.44; N, 8.06; S, 12.29. Found: C, 59.77; $\mathrm{H}, 4.41 ; \mathrm{N}, 8.02 ; \mathrm{S}, 12.21 \%$.

2.22. $\mathrm{N}$-[4-(Aminosulfonyl)phenyl]-2-anilino-4-phenyl-5-[(2oxo-2H-chromen-3-yl)carbonyl]-thiophene-3-carboxamide

(17). Yield (55\%), m.p. $200^{\circ} \mathrm{C}(\mathrm{EtOH})$; IR (KBr) $\nu_{\max }: 3375$, $3261\left(\mathrm{NH}, \mathrm{NH}_{2}\right), 1713(\mathrm{C}=\mathrm{O}), \mathrm{cm}^{-1} ;{ }^{1} \mathrm{H}$ NMR (DMSO-d ${ }_{6}$ ): $\delta$ 6.71-7.27 (m, 7H, Ar- $\mathrm{H}$ and $\left.\mathrm{NH}_{2}\right), 7.29(\mathrm{~d}, 2 \mathrm{H}, J=9 \mathrm{~Hz})$, $7.42-7.59$ (m, 10H, Ar-H), 7.63 (d, 2H, J = 9 Hz), 9.88 (s, 1H, $\mathrm{D}_{2} \mathrm{O}$-exchangeable $\left.\mathrm{NH}\right), 10.02\left(\mathrm{~s}, 1 \mathrm{H}, \mathrm{D}_{2} \mathrm{O}\right.$-exchangeable $\mathrm{NH}) ; \mathrm{MS} m / z$ (\%): $621\left(\mathrm{M}^{+}, 6.8\right), 423$ (100.0), 250 (15.1), 221 (8.2), 199 (8.2), 145 (24.7), 105 (65.8), 77 (87.7). Anal. Calcd 


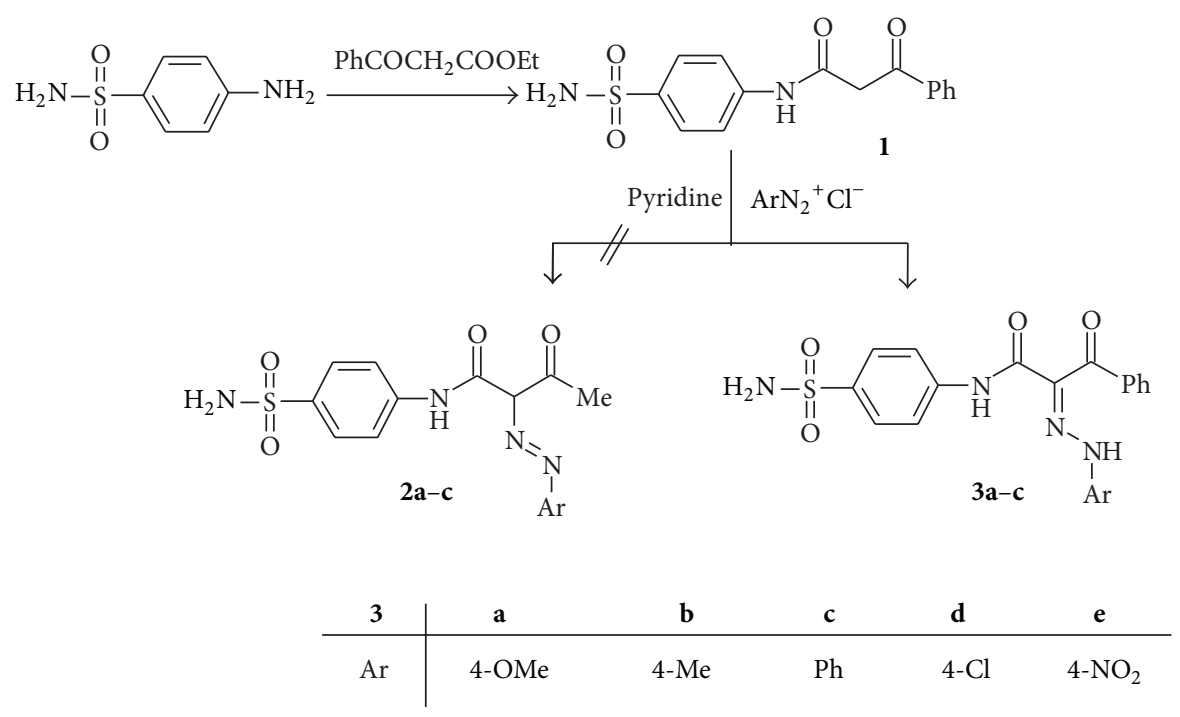

SCHEme 1: Synthesis of hydrazones derivatives.

for $\mathrm{C}_{33} \mathrm{H}_{23} \mathrm{~N}_{3} \mathrm{O}_{6} \mathrm{~S}_{2}$ (621.70): C, 63.76; $\mathrm{H}, 3.73 ; \mathrm{N}, 6.76 ; \mathrm{S}$, 10.32. Found: C, 63.70; H, 3.61; N, 6.67; S, 10.25\%.

2.23. 2-Benzoyl-3-methylsulfanyl-3-phenylamino-N-(4-sulfamoylphenyl)acrylamide (18). Yield (30\%), m.p. $260^{\circ} \mathrm{C}$ (dioxane); IR (KBr) $v_{\max }: 3343,3242\left(\mathrm{NH}, \mathrm{NH}_{2}\right), 1696(\mathrm{C}=\mathrm{O}) \mathrm{cm}^{-1}$; ${ }^{1} \mathrm{H}$ NMR (DMSO-d $\left.)_{6}\right): \delta 3.56\left(\mathrm{~s}, 3 \mathrm{H}, \mathrm{SCH}_{3}\right), 7.24(\mathrm{~d}, 2 \mathrm{H}, J$ $=9 \mathrm{~Hz}), 7.34-7.71\left(\mathrm{~m}, 12 \mathrm{H}, \mathrm{Ar}-\mathrm{H}\right.$ and $\left.\mathrm{NH}_{2}\right), 7.76(\mathrm{~d}, 2 \mathrm{H}, J=$ $9 \mathrm{~Hz}$ ), 10.50 (s, 2H, $\mathrm{D}_{2} \mathrm{O}$-exchangeable $2 \mathrm{NH}$ ); $\mathrm{MS} \mathrm{m} / z(\%)$ : $467\left(\mathrm{M}^{+}, 22.9\right), 296$ (45.7), 197 (25.7), 139 (22.9), 105 (14.3), 92 (54.3), 63 (100). Anal. Calcd for $\mathrm{C}_{23} \mathrm{H}_{21} \mathrm{~N}_{3} \mathrm{O}_{4} \mathrm{~S}_{2}$ (467.56): C, 59.08; H, 4.53; N, 8.99; S, 13.72. Found: C, 59.12; H, 4.50; N, 8.96 ; S, $13.66 \%$.

2.24. 2-(5-Acetyl-3-phenyl-3H-[1,3,4] thiadiazol-2-ylidene)-3oxo-3-phenyl-N-(4-sulfamoylphenyl)propionamide

(20).

Yield (35\%), m.p. $220^{\circ} \mathrm{C}$ (dioxane); IR (KBr) $v_{\max }: 3340$, $3244\left(\mathrm{NH}, \mathrm{NH}_{2}\right), 3061$ (aromatic $\left.\mathrm{CH}\right), 1614(\mathrm{C}=\mathrm{O}) \mathrm{cm}^{-1}$; ${ }^{1} \mathrm{H}$ NMR $\left(\right.$ DMSO-d $\left._{6}\right): \delta 2.49\left(\mathrm{~s}, 3 \mathrm{H}, \mathrm{CH}_{3}\right), 7.15(\mathrm{~s}, 2 \mathrm{H}$, $\mathrm{D}_{2} \mathrm{O}$-exchangeable $\left.\mathrm{NH}_{2}\right), 7.30(\mathrm{~d}, 2 \mathrm{H}, J=9 \mathrm{~Hz}), 7.62(\mathrm{~d}$, $2 \mathrm{H}, J=9 \mathrm{~Hz}), 7.65-8.39$ (m, 10H, Ar-H), 11.95 (s, 1H, D $2 \mathrm{O}-$ exchangeable $\mathrm{NH})$; MS $m / z(\%): 522\left(\mathrm{M}^{+}+2,14.6\right), 520\left(\mathrm{M}^{+}\right.$, 41.6), 320 (27.0), 306 (13.9), 247 (10.2), 214 (7.3), 189 (8.8), 114 (7.3), 105 (20.4), 77 (100.0). Anal. Calcd for $\mathrm{C}_{25} \mathrm{H}_{20} \mathrm{~N}_{4} \mathrm{O}_{5} \mathrm{~S}_{2}$ (520.59): C, 57.68; H, 3.87; N, 10.76; S, 12.32. Found: C, 57.63; $\mathrm{H}, 3.82 ; \mathrm{N}, 10.71 ; \mathrm{S}, 12.29 \%$.

2.25. Agar Diffusion Well Method to Determine the Antimicrobial Activity. The microorganism inoculums were uniformly spread using sterile cotton swab on a sterile Petri dish malt extract agar (for fungi) and nutrient agar (for bacteria). One hundred $\mu \mathrm{L}$ of each sample was added to each well $(10 \mathrm{~mm}$ diameter holes cut in the agar gel, $20 \mathrm{~mm}$ apart from one another). The systems were incubated for 24-48 hours at $37^{\circ} \mathrm{C}$ (for bacteria) and at $28^{\circ} \mathrm{C}$ (for fungi). After incubation, the microorganism's growth was observed. Inhibition of the bacterial and fungal growth was measured in $\mathrm{mm}$. Tests were performed in triplicate [31].

\section{Results and Discussion}

Heterocyclic azo compounds are well known for their use as antineoplastics [32], antidiabetics [33], antiseptics [34], and antibacterial activity [35] and are known to be involved in a number of biological reactions such as inhibition of DNA, RNA, protein synthesis, carcinogenesis, and nitrogen fixation [34-36]. Thus, propionamide 1 was coupled smoothly with diazonium salts, derived from the appropriate aromatic amines [4-methoxyaniline, 4-methylaniline, aniline, 4chloroaniline, and 4-nitroaniline) in pyridine, to afford the respective hydrazones $3 \mathbf{a}-\mathbf{e}$ (Scheme 1). The structures of the products were established on the basis of their elemental analyses and spectral data (IR, ${ }^{1} \mathrm{H}$ NMR, and MS) [see Experimental Part]. In the ${ }^{1} \mathrm{H}$ NMR spectra of compounds $3 \mathbf{a}-\mathbf{e}$, absence of signal assignable to azomethine group $(\mathrm{CH}-$ $\mathrm{N}=\mathrm{N}-$ ) [37] at $\delta 3.00-4.00 \mathrm{ppm}$ ruled out azo form and supported the hydrazone structure of the products.

Further elucidation of the structure of $\mathbf{3 b}$ came from the reaction with malononitrile and triethyl orthformate to furnish the final isolable products corresponding to the 5-cyano-6-imino-4-phenyl-1- $p$-tolyl-1,6-dihydropyridazine3-carboxylic acid (4-sulfamoylphenyl)amide (4) and 4-(6-benzoyl-3-ethoxy-5-oxo-2-p-tolyl-2,3-dihydro-1,2,4triazin-4-(5H)-yl)benzenesulfonamide (5), respectively. The structures of compounds $\mathbf{4}$ and $\mathbf{5}$ were confirmed based on elemental analysis and spectral data studied (Scheme 2 and Experimental Part). Treatment of compound 1 with $\mathrm{N}, \mathrm{N}$-dimethylformamide-dimethylacetal (DMFDMA) in refluxing dry dioxane afforded 2-benzoyl-3dimethylamino- $N$-(4-sulfamoylphenyl)acrylamide (6). The ${ }^{1} \mathrm{H}$ NMR spectrum of compound 6 showed signals at $\delta$ $3.42,8.10$, and 9.98 due to $N, N$-dimethylamino, $\mathrm{C}=\mathrm{CH}-\mathrm{N}$, 


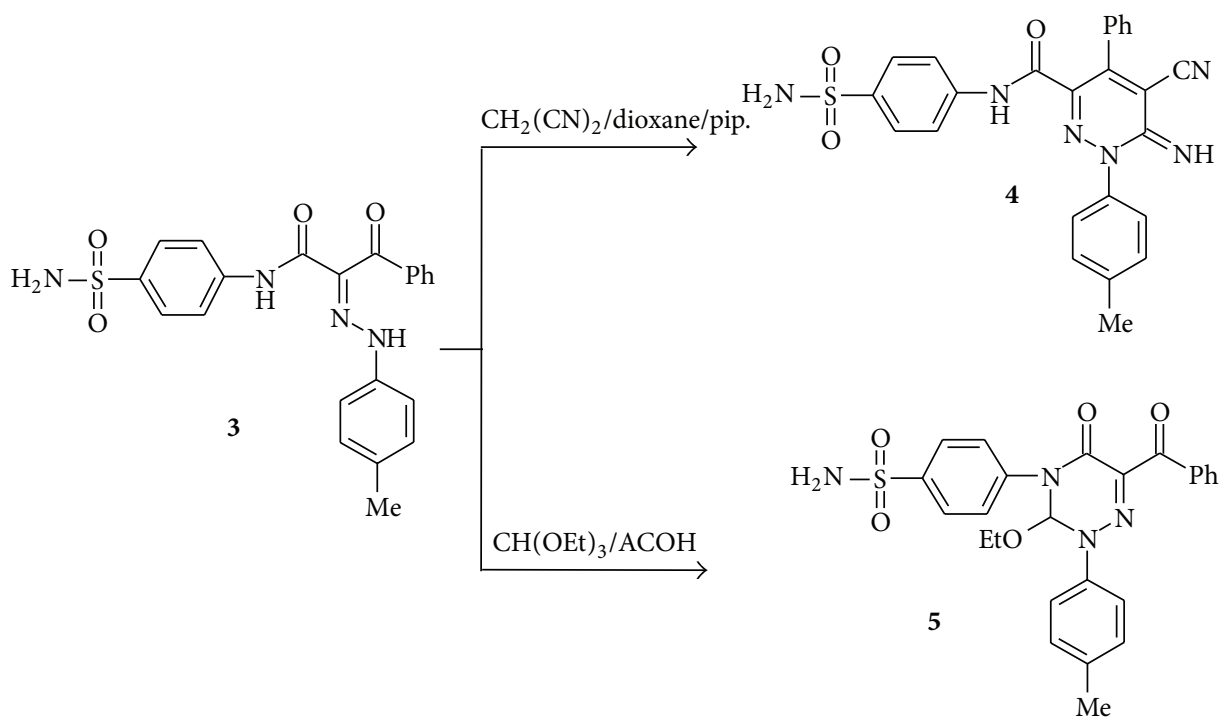

Scheme 2: Synthesis of 1,6-dihydropyridazine and 2,3-dihydro-1,2,4-triazine derivatives.

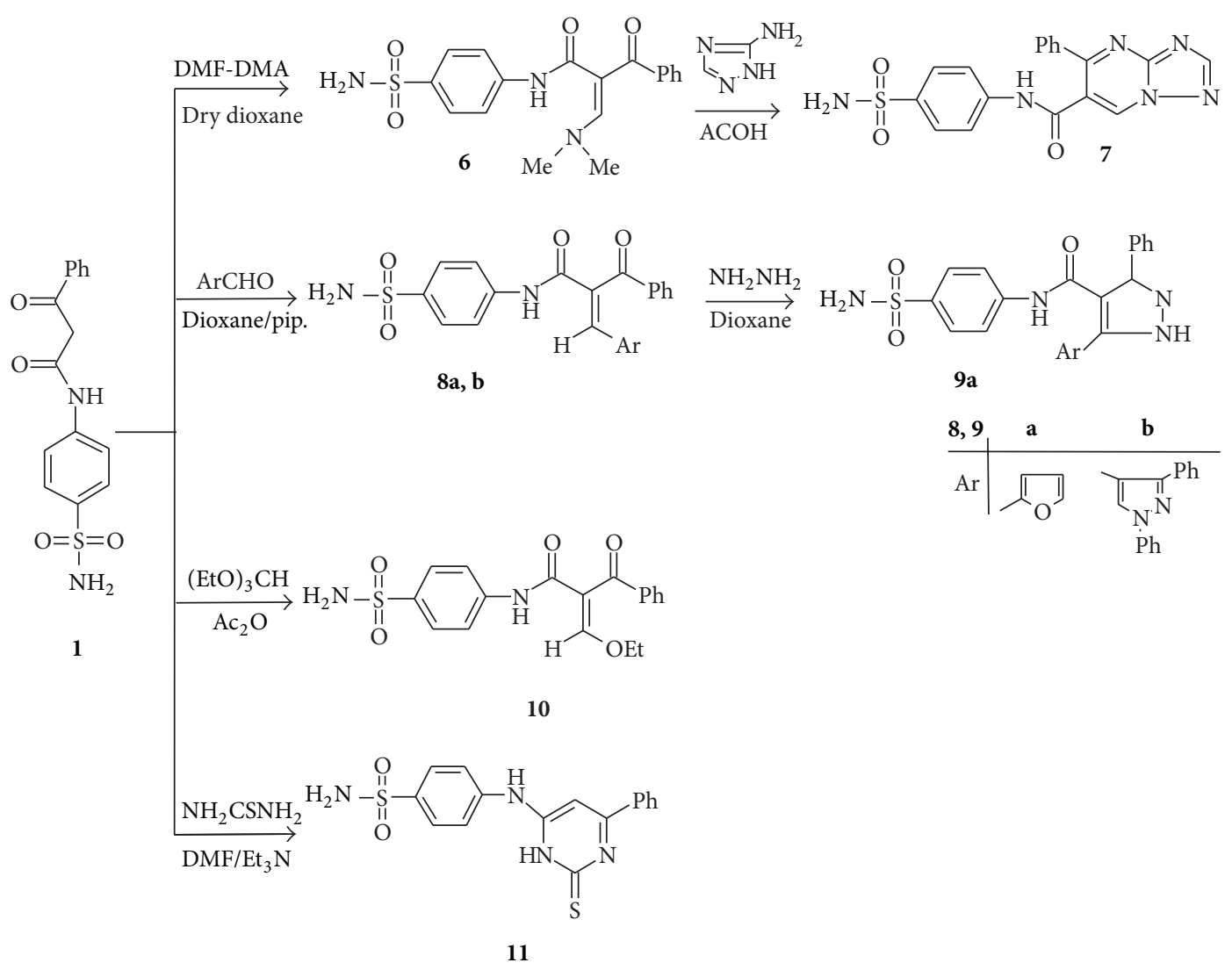

Scheme 3

and amide- $\mathrm{NH}$ protons, respectively. When compound 6 was treated with 3-amino-1,2,4-triazole in acetic acid under reflux this led to formation of 1,2,4-triazolo [1,5-a] pyrimidine derivative 7 (Scheme 3 ). The IR spectrum of the isolated product showed absorption bands at 3261 and $3110 \mathrm{~cm}^{-1}$ characteristic for $\mathrm{NH}$ and $\mathrm{NH}_{2}$ function. Its ${ }^{1} \mathrm{H}$ NMR spectrum showed signals at $\delta 7.68,8.09$, and 8.48 corresponding to triazole- $\mathrm{H}$, pyrimidine- $\mathrm{H}$, and $\mathrm{D}_{2} \mathrm{O}$-exchangeable signal corresponding to $\mathrm{NH}$ protons, respectively. The pathway of formation of product 7 involves 
Michael addition of the exocyclic amino group of the heteroamines to the enaminone double bond of $\mathbf{6}$, followed by in situ tandem elimination of dimethylamine and dehydrative cyclization.

Also, 1 reacts with aromatic aldehydes to afford the corresponding 2-benzoyl-3-aryl-2-yl- $N$-(4-sulfamoylphenyl)acrylamide derivatives $\mathbf{8 a}, \mathbf{b}$ (Scheme 3 ). The IR spectrum of compound $\mathbf{8 a}$, taken as a typical example, revealed absorption bands at 3349,3255 , and $1687 \mathrm{~cm}^{-1}$ corresponding to $\mathrm{NH}, \mathrm{NH}_{2}$, and $\mathrm{CO}$ functions, respectively. Its ${ }^{1} \mathrm{H}$ NMR spectrum showed signals at $\delta 8.48$ and 9.14 corresponding to $\mathrm{CH}$ and $\mathrm{NH}$ protons in addition to aromatic protons at $\delta$ 7.46-7.94. Its mass spectrum showed a molecular ion peak at $m / z$ 396. When the acrylamide derivative 8a was treated with hydrazine hydrate it afforded the corresponding pyrazole derivative 9a (Scheme 3). Spectroscopic data as well as elemental analyses of the obtained products were in complete agreement with the assigned structures 9a.

On the other hand, the reactivity of propionamide $\mathbf{1}$ towards triethylorthoformate and thiourea was investigated. Thus, condensation of $\mathbf{1}$ with triethylorthoformate in refluxing acetic anhydride afforded the ethoxy methylene derivative 10. Establishing of structure $\mathbf{1 0}$ was based on the elemental analysis and spectral data. Treatment of 1 with thiourea afforded the pyrimidine derivative 11. Establishing of compound 11 is based on its elemental analysis and spectral data (IR and ${ }^{1} \mathrm{H}$ NMR) (Scheme 3).

Next, the nucleophilic addition of $\mathbf{1}$ to phenyl isothiocyanate in dimethylformamide, in the presence of potassium hydroxide, afforded the corresponding potassium salt $\mathbf{1 2}$. When the intermediate potassium salt was treated with dilute $\mathrm{HCl}$, it gave the corresponding 2-benzoyl-3-mercapto3-phenylamino- $N$-(4-sulfamoylphenyl)acrylamide (Scheme 4).

The IR spectrum of compound 13 revealed the absorption bands at $3350-3256$ and $1689-1661 \mathrm{~cm}^{-1}$ corresponding to $\mathrm{NH}, \mathrm{NH}_{2}$, and $\mathrm{CO}$ groups, respectively. Its ${ }^{1} \mathrm{H} \mathrm{NMR}$ spectrum showed two $\mathrm{D}_{2} \mathrm{O}$-exchangeable signals at 10.39 , 11.13 , and 12.76 corresponding to $2 \mathrm{NH}$ and $\mathrm{SH}$ proton, respectively. Moreover, the mass spectrum of the product 13 exhibited a molecular ion peak at $\mathrm{m} / z$ 453. Treatment of compound $\mathbf{1 3}$ with 2-bromo-1-phenylethanone in dimethylformamide, in the presence of a catalytic amount of triethylamine, afforded the carboxamide 14 (Scheme 4). The structure of compound $\mathbf{1 4}$ was elucidated from its spectroscopic and elemental analytical data. Thus, it showed absorption bands at $3425-3111$ and $1698-1658 \mathrm{~cm}^{-1}$ due to $\mathrm{NH}, \mathrm{NH}_{2}$, and $\mathrm{CO}$ functions, whereas its ${ }^{1} \mathrm{H}$ NMR spectra revealed two $\mathrm{D}_{2} \mathrm{O}$-exchangeable signals at 9.55 and 9.98 corresponding to $2 \mathrm{NH}$ protons. Heterocyclisation of the intermediate with chloroacetone or ethyl 2-chloroacetate and 3-(2-bromoacetyl)- $2 \mathrm{H}$-chromen-2-one furnished in each case one isolable product (as tested by TLC). The reaction products were identified as $\mathrm{N}$-[4-(aminosulfonyl)phenyl]2-anilino-4-phenylthiophene-5-acetyl-3-carboxami-de (15), 3-phenyl-5-phenylamino-4-(4-sulfa-moylphenyl-carbamoyl)thiophene-2-carboxylic acid ethyl ester (16), and
$\mathrm{N}$-[4-(aminosulfonyl)phenyl]-2-anilino-4-phenyl-5-[(2oxo-2H-chromen-3-yl)carbonyl] thiophene-3-carboxamide (17). The reaction proceeds via nucleophilic displacement of bromide to give $S$-alkylated intermediate followed by loss of water of the latter intermediate to give thiophene derivatives $\mathbf{1 5}$ or $\mathbf{1 6}$ or $\mathbf{1 7}$ as the final products. The structures of the products 15-17 were determined from spectroscopic and elemental analytical data. Thus, compound 15, taken as a typical example, showed absorption bands at 3371, 3289, 3220 , and $1636 \mathrm{~cm}^{-1}$ corresponding to $\mathrm{NH}, \mathrm{NH}_{2}$, and $\mathrm{C}=\mathrm{O}$ groups, respectively. Its ${ }^{1} \mathrm{H}$ NMR spectrum revealed the absence of $\mathrm{CH}_{2}$ protons of chloroacetone and showed signals at $\delta 9.46$ and 9.88 due to $2 \mathrm{NH}$ protons, in addition to an aromatic multiplet in the region $\delta 7.13-7.63$. The ${ }^{13} \mathrm{C}$ NMR of compound 15 revealed signals at 28.15, 162.59, and 189.30 for the carbons of the $\mathrm{CH}_{3}$ of $\left(\mathrm{COCH}_{3}\right), \mathrm{CO}$ of $(\mathrm{CONH})$, and $\mathrm{CO}$ of $\left(\mathrm{COCH}_{3}\right)$ groups.

Furthermore, the nonisolated potassium salt was methylated by treatment with methyl iodide to afford the novel ketene $N, S$-acetal $\mathbf{1 8}$. The structure of the synthesized product was established on the basis of their elemental analysis and spectral data [see the Experimental Part].

Heterocyclisation of the intermediate 12 with 2-oxo$\mathrm{N}$-phenylpropane hydrazonoyl chloride [38] furnished one isolable product (as tested by TLC). The reaction product was identified as 2-(5-acetyl-3-phenyl-3H-[1,3,4]-thiadiazol-2ylidene)-3-oxo-3-phenyl- $N$-(4-sulfamoylphenyl)propionamide (20) (Scheme 4). The structure of the product 20 was determined from spectroscopic and elemental analytical data. Thus, the IR spectrum of compound 20 revealed absorption bands at 3340,3244 , and $1614 \mathrm{~cm}^{-1}$ corresponding to $\mathrm{NH}$, $\mathrm{NH}_{2}$, and $\mathrm{CO}$ groups, respectively. Its ${ }^{1} \mathrm{H}$ NMR spectrum revealed signals at $\delta 2.49$ and 11.95 due to $\mathrm{CH}_{3}$ and $\mathrm{NH}$ protons, in addition to an aromatic multiplet in the region $\delta$ 7.30-8.39. The aforementioned results indicate that the reaction proceeds via $S$-alkylation [39], to give $S$-alkylated intermediate 19 which cyclized in situ under the employed reaction conditions and elimination of aniline molecule gave the desired product 20 (Scheme 4).

Screening for Antimicrobial Activity. The newly synthesized compounds 3a, 3c, 6, 7, 8b, 10, 15, 16, and 17 were evaluated for their in vitro antibacterial activity against Staphylococcus aureus (RCMB-000106) (SA) and Bacillus subtilis (RCMB-000108) (BS) as examples of Gram-positive bacteria and Pseudomonas aeruginosa (RCMB-000102) (PA) and Escherichia coli (RCMB-000103) (EC) as examples of Gram-negative bacteria. They were also evaluated for their in vitro antifungal activity against Aspergillus fumigatus (RCMB-002003) (AF), Saccharomyces cerevisiae (RCMB006002) (SC), and Candida albicans (RCMB-005002) (CA) fungal strains. Inhibition zone diameter (IZD) in $\mathrm{mm}$ was used as criterion for the antimicrobial activity using the agar diffusion well method. The fungicide clotrimazole and the bactericide streptomycin were used as references to evaluate the potency of the tested compounds under the same conditions. The results are depicted in Table 1. From the data given by Table 1 we concluded that most of the 

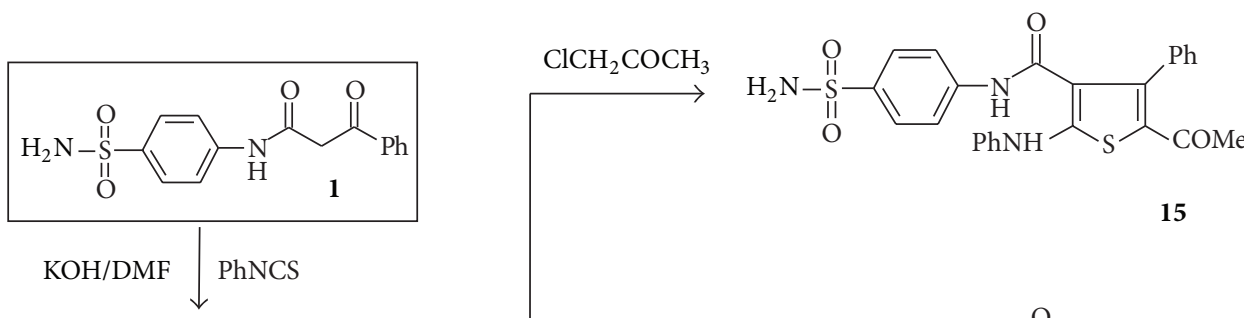

15

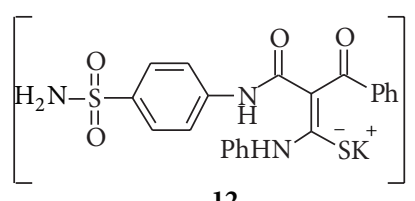

12<smiles>CCOC(=O)c1sc(Nc2ccc(S(N)(=O)=O)cc2)c(C(=O)Nc2ccccc2)c1-c1ccccc1</smiles>

Dil HCl<smiles>NS(=O)(=O)c1ccc(NC(=O)C(C(=O)c2ccccc2)=C(S)c2ccccc2)cc1</smiles><smiles>CCCCCCCCCCCCCCCCCC(=O)O</smiles><smiles>NS(=O)(=O)c1ccc(NC(=O)c2c(Nc3ccccc3)sc(C(=O)c3ccccc3)c2-c2ccccc2)cc1</smiles>

14

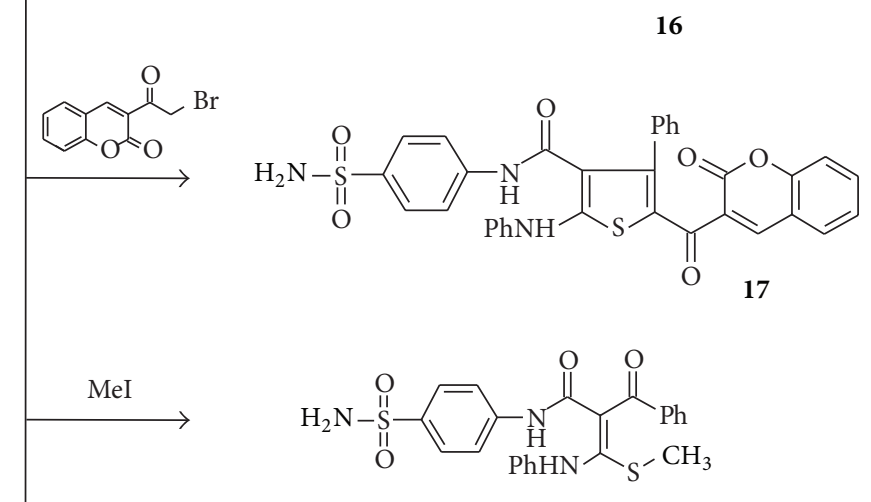

18
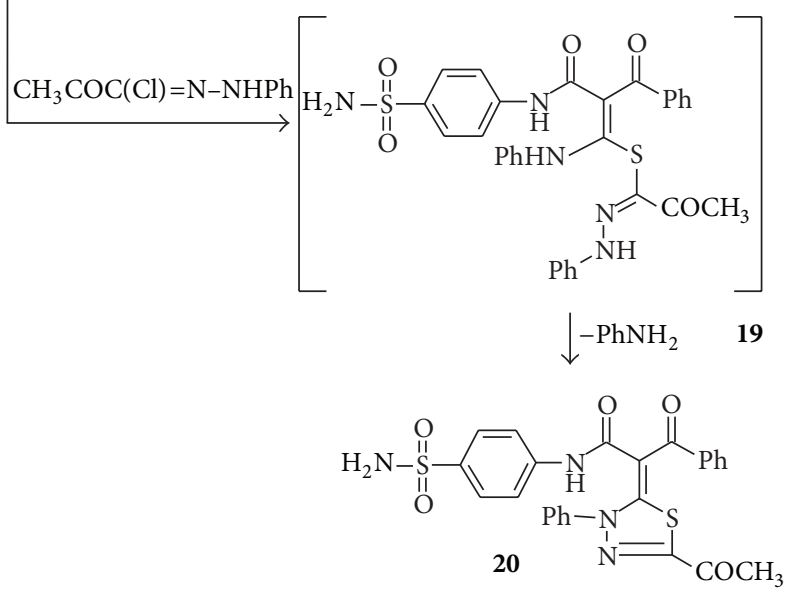

SCHEME 4

tested compounds displayed variable degrees of antibacterial activity against Gram-positive bacteria, Gram-negative bacteria strains, and also against fungal strains in comparison to the standard in each case which revealed that these compounds are biologically active. Compound 7 exhibited high degree of antibacterial activity against Gram-positive bacteria $(S A)$. Compounds $3 \mathbf{c}$ and 7 have moderate degree of antibacterial activity against Gram-negative bacteria $(E C)$ and $(P A)$. All the compounds exhibited moderate antifungal activity against $(A F)$ and high activity against $(S C, C A)$. The structure antimicrobial activity relationship of the synthesized compounds revealed that the maximum activity was attained with compound 15, having acetyl thiophene moiety.

\section{Conclusions}

Several new hydrazones, pyridazines, triazines, acrylamides, pyrazoles, triazolo[1,5-a]pyrimidines, thiadiazoles, dihydropyrimidines, and thiophenes containing sulfamoyl moiety were prepared using simple methods via a versatile; readily accessible 3-oxo-3-phenyl- $N$-(4-sulfamoylphenyl)propionamide (1) is demonstrated. The structures of the 
Table 1: Antimicrobial and Antifungal Activities of the Synthesized Compounds.

\begin{tabular}{|c|c|c|c|c|c|c|c|}
\hline \multirow{3}{*}{ Sample no. } & \multicolumn{7}{|c|}{ Inhibition zone diameter (mm) } \\
\hline & \multicolumn{2}{|c|}{ Gram (-) } & \multicolumn{2}{|c|}{ Gram (+) } & \multicolumn{3}{|c|}{ Fungi } \\
\hline & $(\mathrm{PA})$ & (EC) & $(\mathrm{SA})$ & (BS) & $(\mathrm{AF})$ & $(\mathrm{SC})$ & $(\mathrm{CA})$ \\
\hline $3 a$ & $10.7 \pm 0.3$ & $10.1 \pm 0.4$ & $12.2 \pm 0.3$ & $11.1 \pm 0.4$ & $11.9 \pm 0.4$ & $12.5 \pm 0.4$ & $13.4 \pm 0.4$ \\
\hline $3 c$ & $12.9 \pm 0.4$ & $11.7 \pm 0.4$ & $12.1 \pm 0.4$ & $12.3 \pm 0.4$ & $18.7 \pm 0.4$ & $20.8 \pm 0.6$ & $14.7 \pm 0.5$ \\
\hline 6 & $10.6 \pm 0.3$ & $10.8 \pm 0.4$ & $12.2 \pm 0.4$ & $11.3 \pm 0.4$ & $17.1 \pm 0.5$ & $17.9 \pm 0.6$ & $14.8 \pm 0.4$ \\
\hline 7 & $13.4 \pm 0.4$ & $13.1 \pm 0.4$ & $19.7 \pm 0.4$ & $20.1 \pm 0.5$ & $17.6 \pm 0.4$ & $18.8 \pm 0.5$ & $13.9 \pm 0.5$ \\
\hline $8 \mathbf{b}$ & $11.1 \pm 0.3$ & $12.7 \pm 0.4$ & $11.8 \pm 0.3$ & $11.5 \pm 0.4$ & $15.3 \pm 0.5$ & $19.1 \pm 0.4$ & $15.2 \pm 0.5$ \\
\hline 10 & $9.7 \pm 0.2$ & $8.9 \pm 0.4$ & $11.2 \pm 0.4$ & $10.8 \pm 0.4$ & $12.1 \pm 0.5$ & $11.9 \pm 0.4$ & $11.7 \pm 0.5$ \\
\hline 15 & $24.3 \pm 0.1$ & $25.6 \pm 0.1$ & $25.1 \pm 0.5$ & $30.1 \pm 0.6$ & $26.1 \pm 0.5$ & $23.1 \pm 0.4$ & $18.3 \pm 0.6$ \\
\hline 16 & $11.1 \pm 0.4$ & $10.3 \pm 0.3$ & $9.8 \pm 0.3$ & $10.9 \pm 0.4$ & $11.4 \pm 0.3$ & $12.2 \pm 0.4$ & $11.3 \pm 0.5$ \\
\hline 17 & $9.2 \pm 0.3$ & $7.8 \pm 0.4$ & $8.9 \pm 0.3$ & $9.7 \pm 0.3$ & $10.5 \pm 0.4$ & $10.3 \pm 0.4$ & $9.5 \pm 0.4$ \\
\hline Clotrimazole & - & - & - & - & $26.1 \pm 0.5$ & $23.1 \pm 0.4$ & $18.3 \pm 0.6$ \\
\hline Streptomycin & - & - & $25.1 \pm 0.5$ & $30.1 \pm 0.6$ & - & - & - \\
\hline Streptomycin & $24.3 \pm 0.4$ & $25.6 \pm 0.5$ & - & - & - & - & - \\
\hline
\end{tabular}

Data are expressed in the form of mean \pm SD. Mean zone of inhibition in $\mathrm{mm} \pm$ Standard deviation beyond well diameter (6 mm) produced on a range of environmental and clinically pathogenic microorganism using $(5 \mathrm{mg} / \mathrm{mL})$ concentration of tested sample (100 $\mu \mathrm{L}$ was tested).

newly synthesized compounds were proven by spectral methods and they were tested for their antimicrobial activities. Most of these compounds showed promising activities against both Gram-positive, Gram-negative bacteria and fungi.

\section{Conflict of Interests}

The author declares that there is no conflict of interests regarding the publication of this paper.

\section{Acknowledgment}

The support from Chemistry Department, Faculty of Science, Cairo University, is gratefully acknowledged.

\section{References}

[1] M. S. A. El-Gaby, A. A. Atalla, A. M. Gaber, and K. A. Abd Al-Wahab, "Studies on aminopyrazoles: antibacterial activity of some novel pyrazolo[1,5-a]pyrimidines containing sulfonamido moieties," Farmaco, vol. 55, no. 9-10, pp. 596-602, 2000.

[2] M. S. A. El-Gaby, N. M. Taha, J. A. Micky, and M. A. M. S. El-Sharief, "Preparation of some novel 3,5-diaminopyrazole, pyrazolo[1,5-a] $[1,3,5]$ triazine and pyrazolo[1,5-a]-pyrimidine derivatives containing sulfonamido moieties as antimicrobial agents," Acta Chimica Slovenica, vol. 49, no. 1, pp. 159-171, 2002.

[3] M. E. Azab, M. M. Youssef, and E. A. El-Bordany, "synthesis and antibacterial evaluation of novel heterocyclic compounds containing a sulfonamido moiety," Molecules, vol. 18, pp. 832844, 2013.

[4] M. S. A. El-Gaby, A. M. Gaber, A. A. Atalla, and K. A. Abd AlWahab, "Novel synthesis and antifungal activity of pyrrole and pyrrolo[2,3-d]pyrimidine derivatives containing sulfonamido moieties," Farmaco, vol. 57, no. 8, pp. 613-617, 2002.

[5] T. H. Maren, "Relatons between structure and biological activity of sulfonamides," Annual Review of Pharmacology and Toxicology, vol. 16, pp. 309-327, 1976.
[6] C. T. Supuran, A. Scozzafava, B. C. Jurca, and M. A. Ilies, "Carbonic anhydrase inhibitors-part 49: synthesis of substituted ureido and thioureido derivatives of aromatic/heterocyclic sulfonamides with increased affinities for isozyme I," European Journal of Medicinal Chemistry, vol. 33, no. 2, pp. 83-93, 1998.

[7] J. J. Li, G. D. Anderson, E. G. Burton et al., "1,2Diarylcyclopentenes as selective cyclooxygenase-2 inhibitors and orally active anti-inflammatory agents," Journal of Medicinal Chemistry, vol. 38, no. 22, pp. 4570-4578, 1995.

[8] H. Yoshino, N. Ueda, J. Niijima et al., "Novel sulfonamides as potential, systemically active antitumor agents," Journal of Medicinal Chemistry, vol. 35, no. 13, pp. 2496-2497, 1992.

[9] M. T. Labro, "Immunomodulation by antibacterial agents: is it clinically relevant?” Drugs, vol. 45, no. 3, pp. 319-328, 1993.

[10] Y. Rajendra Prasad, A. Lakshmana Rao, L. Prasoona, K. Murali, and P. Ravi Kumar, "Synthesis and antidepressant activity of some 1,3,5-triphenyl-2-pyrazolines and 3-(2"-hydroxy naphthalen-1" $1^{\prime \prime}$ yl)-1,5-diphenyl-2-pyrazolines," Bioorganic and Medicinal Chemistry Letters, vol. 15, no. 22, pp. 5030-5034, 2005.

[11] Z. Özdemir, H. B. Kandilci, B. Gümüşel, Ü. Çaliş, and A. A. Bilgin, "Synthesis and studies on antidepressant and anticonvulsant activities of some 3-(2-furyl)-pyrazoline derivatives," European Journal of Medicinal Chemistry, vol. 42, no. 3, pp. 373379, 2007.

[12] A. Özdemir, G. Turan-Zitouni, Z. Asim Kaplancikli, G. Revial, and K. Güven, "Synthesis and antimicrobial activity of 1-(4aryl-2-thiazolyl)-3-(2-thienyl)-5-aryl-2-pyrazoline derivatives," European Journal of Medicinal Chemistry, vol. 42, no. 3, pp. 403409, 2007.

[13] A. Gürsoy, Ş. Demirayak, G. Çapan, K. Erol, and K. Vural, "Synthesis and preliminary evaluation of new 5-pyrazolinone derivatives as analgesic agents," European Journal of Medicinal Chemistry, vol. 35, no. 3, pp. 359-364, 2000.

[14] Z. Brzozowski, F. S,czewski, and M. Gdaniec, "Synthesis, structural characterization and antitumor activity of novel 2,4diamino-1,3,5-triazine derivatives," European Journal of Medicinal Chemistry, vol. 35, no. 12, pp. 1053-1064, 2000.

[15] C. S. Konkoy, D. B. Fick, S. X. Cai, N. C. Lan, and J. F. W. Keana, "Substituted 5-oxo-5,6,7,8-tetrahydro-4H-1-benzopyrans and 
benzothiopyrans and the use thereof as potentiators of AMPA," Chemical Abstracts, vol. 134, Article ID 29313a, 2001, International Application WO 00/75123 A1, 2000.

[16] F. Giordanetto, O. Karlsson, J. Lindberg et al., "Discovery of cyclopentane- and cyclohexane-trans-1,3-diamines as potent melanin-concentrating hormone receptor 1 antagonists," Bioorganic and Medicinal Chemistry Letters, vol. 17, no. 15, pp. 52225231, 2007.

[17] M. K. Parai, G. Panda, V. Chaturvedi, Y. K. Manju, and S. Sinha, "Thiophene containing triarylmethanes as antitubercular agents," Bioorganic and Medicinal Chemistry Letters, vol. 18, no. 1, pp. 289-292, 2008.

[18] W. W. Wardakhan, O. M. E. Abdel-Salam, and G. A. Elmegeed, "Screening for antidepressant, sedative and analgesic activities of novel fused thiophene derivatives," Acta Pharmaceutica, vol. 58, no. 1, pp. 1-14, 2008.

[19] P. R. Kumar, S. Raju, P. Satish Goud et al., "Synthesis and biological evaluation of thiophene [3,2-b] pyrrole derivatives as potential anti-inflammatory agents," Bioorganic and Medicinal Chemistry, vol. 12, no. 5, pp. 1221-1230, 2004.

[20] C. Bonini, L. Chiummiento, M. de Bonis et al., "Synthesis, biological activity and modelling studies of two novel anti HIV PR inhibitors with a thiophene containing hydroxyethylamino core," Tetrahedron, vol. 61, no. 27, pp. 6580-6589, 2005.

[21] L. Brault, E. Migianu, A. Néguesque, E. Battaglia, D. Bagrel, and G. Kirsch, "New thiophene analogues of kenpaullone: synthesis and biological evaluation in breast cancer cells," European Journal of Medicinal Chemistry, vol. 40, no. 8, pp. 757-763, 2005.

[22] E. S. Darwish, "Facile synthesis of heterocycles via 2picolinium bromide and antimicrobial activities of the products," Molecules, vol. 13, no. 5, pp. 1066-1078, 2008.

[23] E. S. Darwish, N. A. Kheder, and A. M. Farag, "Synthesis and antimicrobial evaluation of some new pyridine based heterocycles," Heterocycles, vol. 81, no. 10, pp. 2247-2256, 2010.

[24] E. S. Darwish, A. M. Abdel Fattah, F. A. Attaby, and O. N. AlShayea, "Synthesis and antimicrobial evaluation of some novel thiazole, pyridone, pyrazole, chromene, hydrazone derivatives bearing a biologically active sulfonamide moiety," International Journal of Molecular Sciences, vol. 15, pp. 1237-1254, 2014.

[25] E. S. Darwish, K. A. Atia, and A. M. Farag, "Synthesis and antimicrobial evaluation of some isoxazole based heterocycles," Heterocycle, vol. 89, no. 6, pp. 1393-1411, 2014.

[26] J. Tang, L. M. Shewchuk, H. Sato, M. Hasegawa, Y. Washio, and N. Nishigaki, "Anilinopyrazole as selective CDK2 inhibitors: design, synthesis, biological evaluation, and X-ray crystallographic analysis," Bioorganic and Medicinal Chemistry Letters, vol. 13, no. 18, pp. 2985-2988, 2003.

[27] M. S. T. Makki, R. M. Abdel-Rahman, and M. El-Shahawi, "Synthesis of polyfunctional organic systems containing 1acyl/aroyl-anilido-butadienes and their uses as photochemical probes and chelating agents for removal of bismuth (III) from industrial wastewater," Journal of Bioanalysis and Biomedicine, vol. 2, no. 4, pp. 85-90, 2010.

[28] C. F. Koelsch, "Bromination of 3-acetocoumarin," Journal of the American Chemical Society, vol. 72, no. 7, pp. 2993-2995, 1950.

[29] P. Czerney and H. Hartman, "3- $\alpha$-bromacetyl-coumarines as synthones for heterocyclic substituted coumarines," Journal für Praktische Chemie, vol. 325, pp. 551-560, 1983.

[30] R. N. Butler, "The diazotization of heterocyclic primary amines," Chemical Reviews, vol. 75, no. 2, pp. 241-257, 1975.
[31] E. F. A. Smania, F. D. Monache, A. Smania Jr., R. A. Yunes, and R. S. Cuneo, "Triterpenes and sterols from ganoderma australe (Fr) Pat. (Aphyllophoromycetideae)," International Journal of Medicinal Mushrooms, vol. 1, pp. 325-334, 1999.

[32] R. G. Child, R. G. Wilkinson, and A. Tomcu-Fucik, "effect of substrate orientation of the adhesion of polymer joints," Chemical Abstracts, vol. 87, p. 6031, 1977.

[33] H. G. Garg and C. Prakash, "Potential antidiabetics, 11. Preparation of 4-arylazo-3,5-disubstituted-(2H)-1,2,6-thiadiazine 1,1dioxides," Journal of Medicinal Chemistry, vol. 15, no. 4, pp. 435436, 1972.

[34] C. H. Browing, J. B. Cohen, S. Ellingworth, and R. Gulbransen, "The antiseptic properties of the aminoderivatives of styryl and anil quinoline," Journal Storage, vol. 100, pp. 293-325, 1926.

[35] G. Swati, K. Romila, I. K. Sharma, and P. S. Verma, "Synthesis, characterisation and antimicrobial screening of some azo compounds," International Journal of Applied Biology and Pharmaceutical Technology, vol. 2, pp. 332-338, 2011.

[36] R. N. Goyal, M. S. Verma, and N. K. Singhal, "Voltammetric investigations of the reduction of direct orange-31 a bisazo dye," Croatica Chemica Acta, vol. 71, no. 3, pp. 715-726, 1998.

[37] M. C. Hamzacebi, S. Rollas, S. G. Küçükgüzel, and B. K. Kaymakçığlu, "Synthesis and structure elucidation of hydrazones derived from N-(2,4-dimethylphenyl)-3oxobutanamide," Arkivoc, vol. 12, pp. 188-194, 2008.

[38] N. F. Eweiss and A. Osman, "Synthesis of heterocycles-part II. New routes to acetylthiadiazolines and alkylazothiazoles," Journal of Heterocyclic Chemistry, vol. 17, pp. 1713-1717, 1980.

[39] A. A. Geies, A. M. Kamal-Eldeen, A. A. Abdelhafez, and A. M. Gaber, "Synthesis of some thiazolo(3,2-a) pyrimidines," Phosphorus, Sulfur, and Silicon and the Related Elements, vol. 56, pp. 87-93, 1991. 

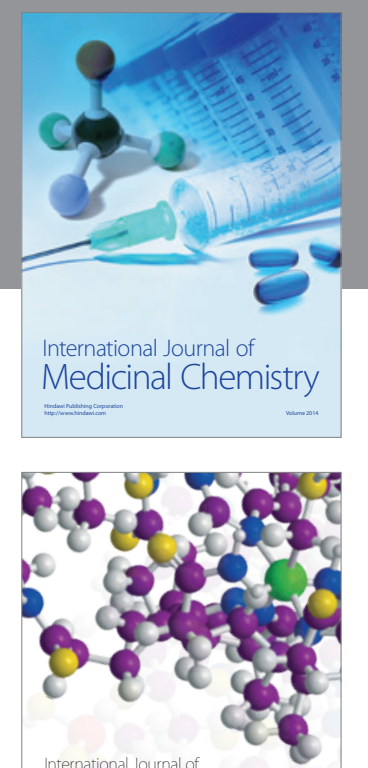

\section{Carbohydrate} Chemistry

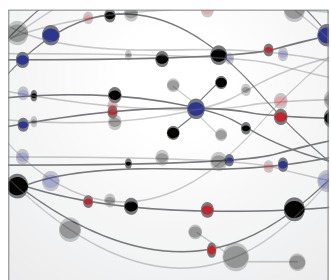

The Scientific World Journal
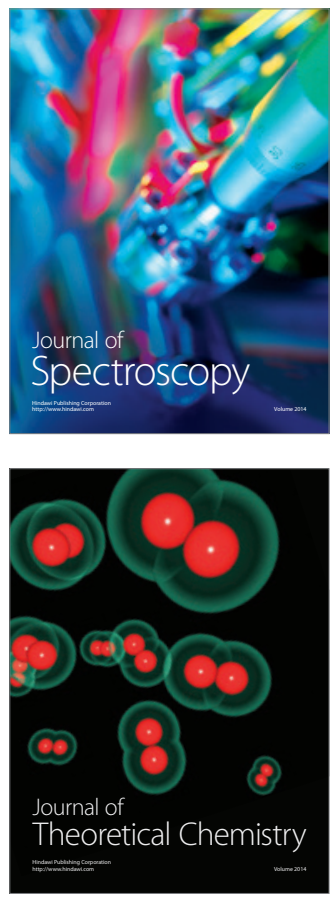
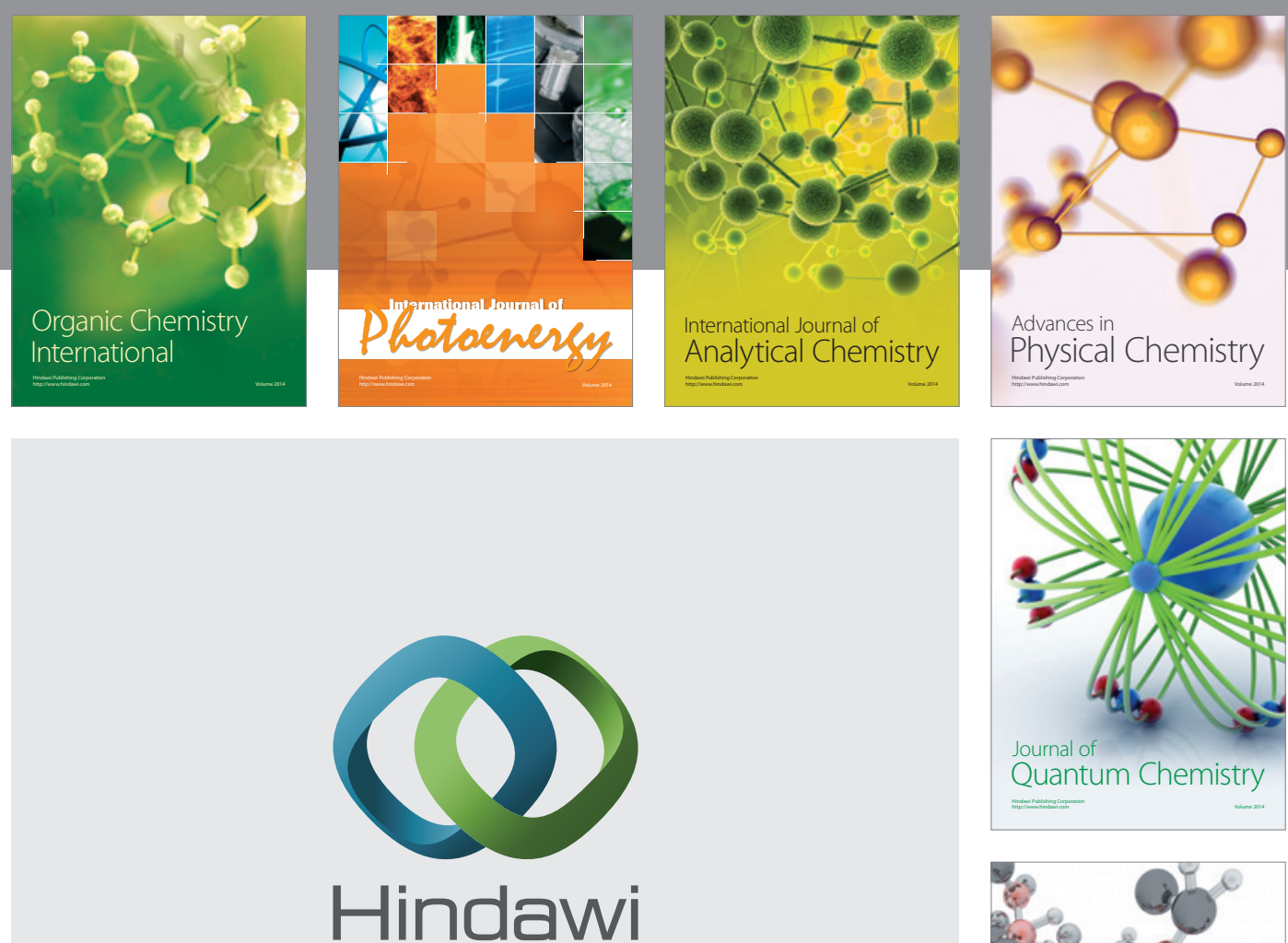

Submit your manuscripts at

http://www.hindawi.com

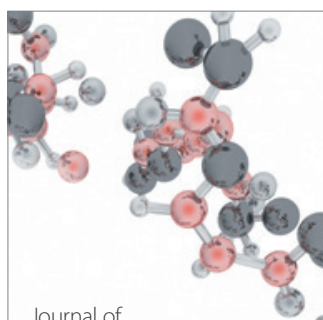

Analytical Methods

in Chemistry

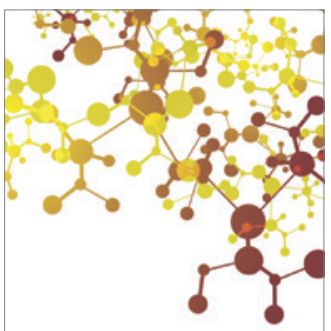

Journal of

Applied Chemistry

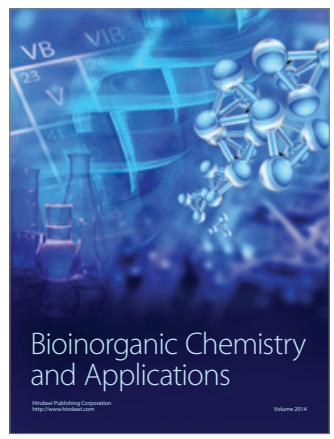

Inorganic Chemistry
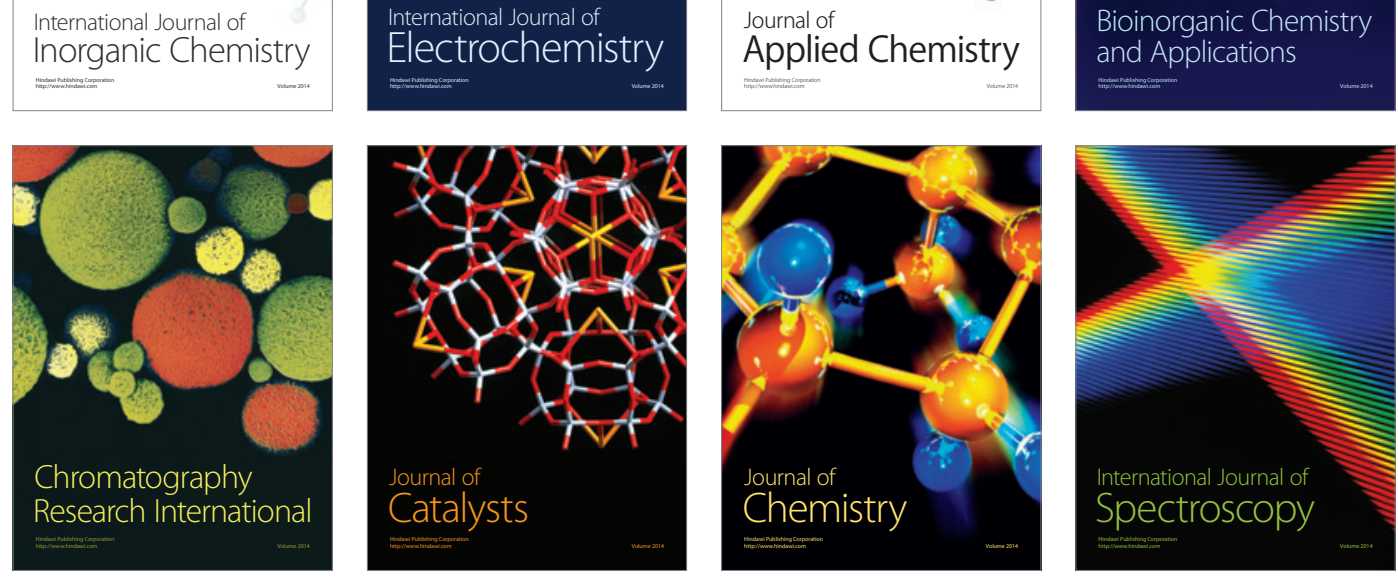